\title{
Mathematical Explanations in Evolutionary Biology or Naturalism? A Challenge for the Statisticalist
}

\author{
Fabio Sterpetti ${ }^{1}$ id
}

Accepted: 1 September 2021 / Published online: 23 September 2021

(C) The Author(s) 2021

\begin{abstract}
This article presents a challenge that those philosophers who deny the causal interpretation of explanations provided by population genetics might have to address. Indeed, some philosophers, known as statisticalists, claim that the concept of natural selection is statistical in character and cannot be construed in causal terms. On the contrary, other philosophers, known as causalists, argue against the statistical view and support the causal interpretation of natural selection. The problem I am concerned with here arises for the statisticalists because the debate on the nature of natural selection intersects the debate on whether mathematical explanations of empirical facts are genuine scientific explanations. I argue that if the explanations provided by population genetics are regarded by the statisticalists as non-causal explanations of that kind, then statisticalism risks being incompatible with a naturalist stance. The statisticalist faces a dilemma: either she maintains statisticalism but has to renounce naturalism; or she maintains naturalism but has to content herself with an account of the explanations provided by population genetics that she deems unsatisfactory. This challenge is relevant to the statisticalists because many of them see themselves as naturalists.
\end{abstract}

Keywords Causalism - Counterfactual theory of explanation - Mathematical explanations of natural phenomena $\cdot$ Naturalism $\cdot$ Population genetics explanations $\cdot$ Statisticalism

\section{Introduction}

This article presents a challenge that those philosophers who deny the causal interpretation of explanations provided by population genetics might have to address, a challenge that has so far gone unnoticed. Indeed, some philosophers, known as statisticalists, claim that the concept of natural selection, as it is normally presented in population genetics, is statistical in character and cannot be construed in causal terms (Matthen \& Ariew, 2009; Walsh et al., 2002; Walsh et al., 2017). On the contrary, other philosophers, known as causalists, argue against the statistical view and support the causal interpretation of natural selection (Millstein et al., 2009; Otsuka, 2016; Sober, 1984).

Fabio Sterpetti

fabio.sterpetti@uniroma1.it

1 Sapienza University of Rome, Via Carlo Fea 2, 00161 Rome (RM), Italy 
The problem I focus on here arises for the statisticalists because the debate on the nature of natural selection intersects the debate on whether mathematical explanations of empirical facts are genuine scientific explanations. Indeed, if on the one hand, those philosophers of biology who embrace statisticalism claim that the causal interpretation of natural selection is inadequate, on the other hand, those philosophers of science who support the claim that there are genuine non-causal explanations in science use examples taken from evolutionary biology in order to defend their view (Baron, 2014; Lange, 2013a; Rice, 2015). Those two positions seem thus to reinforce each other. But the relationship between statisticalism and the debate over non-causal explanations implies that statisticalists have to deal with the difficulties the supporters of non-causal explanations have to deal with. One of those difficulties is to provide reasons to think that (at least some kinds of) non-causal explanations are compatible with a naturalist stance. Clearly this is a difficulty only for those philosophers who think of themselves as naturalists, so in a sense its scope is limited. I will argue that if one regards the explanations provided by population genetics as non-causal explanations of a certain kind, then one risks being unable to take a naturalist stance. Despite its scope is quite limited, this challenge is potentially very relevant to the statisticalists, because many of them see themselves as naturalists, and usually do not move their criticisms against the causalist view for any sort of anti-naturalist commitment.

This article does not aim at taking side on the debate between causalists and statisticalists. Rather, the point I wish to make is just that there is a tension between accepting statisticalism and accepting naturalism: either one accepts statisticalism because one thinks that it is a more adequate account of the explanations provided by population genetics, but then one has to renounce naturalism; or one rejects statisticalism because it is not compatible with naturalism, but then one might be left with an unsatisfactory account of the explanations provided by population genetics, a field that is crucial for every naturalist view on knowledge to be defined as such, given the prominent role that evolutionism plays in the development of any naturalist stance (Giere, 2006) and the centrality of population genetics to evolutionary biology.

The structure of the paper is as follows: in Sect. 2, the statisticalist view is presented; in Sect. 3, the issue of non-causal explanations in science is introduced; in Sect. 4, a relevant kind of non-causal, mathematical explanations is presented; in Sect. 5, it is discussed whether that kind of non-causal, mathematical explanations is compatible with a naturalist stance; in Sect. 6, three objections to the claim that that kind of non-causal, mathematical explanations is incompatible with a naturalist stance are considered; in Sect. 7, statistical explanations in population genetics are discussed; in Sect. 8, Walsh's counterfactual account of statistical explanations in population genetics is discussed; finally, in Sect. 9, some conclusions are drawn.

\section{Statisticalism}

To illustrate the statisticalist view, it may be useful to firstly present the view the statisticalists oppose to, namely the causalist view. A well-known formulation of the causalist view rests on the so-called Force Analogy (see, e.g., Luque, 2016; Hitchcock \& Velasco, 2014). The idea is to present evolution as if it were driven by forces that behave analogously to the forces in Newtonian mechanics. The most developed use of the Force Analogy to illustrate evolutionary theory is made by Sober in his book The Nature of Selection. According to Sober, in "evolutionary theory, the forces of mutation, migration, selection and drift 
constitute causes that propel a population through a sequence of gene frequencies. To identify the causes of the current state [...] requires describing which evolutionary forces impinged" (Sober, 1984, p. 141). The Force Analogy mainly rests on the idea that as forces in mechanics are usually taken to be causes of motion, so evolutionary forces in population genetics should be taken to be causes of change in gene frequencies.

Contrary to this view, the statisticalists think that the claim that evolutionary forces modify gene frequencies directly implies a disputable ontological commitment. For example, Sober holds that natural selection is evolution due to heritable variation in fitness. ${ }^{1}$ In this line of reasoning, an evolutionary force such as selection would not be reducible to the sequence of events that lead from variation in heritable fitness to a new gene frequency, it should rather be understood as a force that appears when organisms have heritable differences that lead to differential reproductive success (Matthen \& Ariew, 2009). In this view, evolution does not occur merely because types of organism reproduce and die in differing amounts. Rather evolution is driven by natural selection. If selection is a force in the same way in which a Newtonian force is a force, then we should assume that it is natural selection that acts on gene frequencies. According to the statisticalists, this construal of selection is unacceptable, since it implies a sort of reification of the concept of natural selection, which illegitimately leads to an inflation of our ontology (ibidem). No extra cause is needed to account for how gene frequencies change at the population level besides those natural events that are responsible for the survival or the death of organisms at the individual level. So, according to the statisticalists, the Force Analogy is unacceptable because natural selection in population genetics does not really cause any variance in gene frequencies. Natural selection is just a statistical abstraction of the events that are the real causes of gene frequencies, namely births, deaths, and matings, which occur at the individual level.

Another fiercely debated issue by causalists and statisticalists is the nature of drift. Indeed, the Force Analogy seems to imply that evolutionary forces are distinct forces, i.e., forces amenable to be separately defined and investigated. The difficulty of supporting such claim becomes particularly evident when one considers drift. The question is: Is drift really an independent cause of evolution? More precisely: Can drift really be distinguished from natural selection? If one accepts the causalist view, each evolutionary force has to be regarded as an independent 'fact of nature'. On the contrary, if one adopts statisticalism, one cannot neatly distinguish natural selection from drift. Indeed, drift is usually understood as departures from expected gene frequencies that arise in finite populations. Briefly, when one kind of individuals is better endowed than another for reproduction, from a statistical viewpoint, one might expect that the better endowed kind will increase, but this expectation can be frustrated by actual outcomes. Departures from expected values are what population geneticists call drift (Matthen, 2010).

An analogy with coin tossing may be of use to illuminate random drift. If one has a fair coin and one tosses it ten times, the expected outcome is 5 heads and 5 tails; but it well may be the case that one gets 6 heads and 4 tails or 8 heads and 2 tails. If one increases the number of tosses (say one tosses the coin 1,000 times), the frequencies obtained will approximate to the expected outcome. If one increases further the number of tosses (say

\footnotetext{
${ }^{1}$ Cf., e.g., Sober (1984), p. 23: "So we should formulate the Darwinian idea first by saying that evolution by the natural selection of organisms will occur only if organisms exhibit heritable variation in fitness. And [...] we can say that this necessary condition will also be sufficient, if no other evolutionary force intrudes." Cf. also Matthen \& Ariew (2009), p. 204: "we assume Sober's fairly orthodox treatment (1984, 21-22): natural selection is evolution due to heritable variation in fitness."
} 
one tosses the coin 1,000,000 times), the frequencies obtained will approximate to the expected outcome even better. So, we can say that the magnitude of this sampling error, i.e., drift, is inversely proportional to sample size: the smaller the population, the bigger the deviation from the expected outcome.

How should one explain drift? For the causalists, drift is a natural fact or process in which heritable differences between entities are causally irrelevant to differences in reproductive success. For instance, Stephens (2004) suggests that drift acts on populations, causing sampling error. In this view, drift is a sort of force with a greater strength in small populations. Consider again the coin tosses example: according to Stephens, drift "plays a larger role in flipping a fair coin 10 times than it does in flipping a coin 10,000 times" (Stephens, 2004, p. 556). On the contrary, according to the statisticalists, drift is explained by a theorem of probability theory, namely the law of large numbers, according to which the results obtained from a large number of trials tend to become closer to the expected value as more trials are performed (Walsh et al., 2002). For the statisticalists, drift is departures from expected values attributable to the statistical uncertainty that is inherent in any series of births, deaths, and matings. Drift is not a separate natural process over and above the individual-level causes of such events. No extra cause is needed to account for the departures from expected values. The only relevant feature in drift explanations is population size. And, in this perspective, it is just this feature that distinguishes selection explanations from drift explanations:

Natural selection is a statistical trend in the accumulation of trials [...]. Such statistical trends admit of a variety of actual outcomes. In an infinite series of trials, the outcome is certain; however, in a finite series of trials, there is a spread of possible outcomes, each with its own probability. Drift is simply the uncertainty associated with this spread of possibilities. Natural selection and drift are not distinct processes working on a finite population, but mathematically connected aspects of the same accumulation. (Matthen, 2009, p. 484).

Now, let us concede to the statisticalist, at least for the sake of the argument, that her interpretation of population genetics explanations has to be preferred because it does not posit any superfluous cause, and so it does not inflate our ontology without reason. According to this view, the statisticalist interpretation of population genetics explanations has to be preferred because it is more ontologically parsimonious than the causal interpretation, and ontological parsimony is a desirable epistemic virtue, so that, other things being equal, we should always prefer the more ontologically parsimonious interpretation among rival interpretations of a given phenomenon (Baker, 2016). For example, Walsh (2007) rephrases a famous sentence by Newton and states that, as far as "hypostatizing selection and drift goes, statisticalism holds that 'more is in vain when less will serve; for [statisticalism] is pleased with simplicity, and effects not the pomp of superfluous causes'." (Walsh, 2007, p. 301). Here I am not interested in discussing whether the statistical interpretation is really able to explain the same scope of things that the causal interpretation is able to explain without positing any cause at the population level, a claim disputed by causalists, so that the 'other things being equal' clause is satisfied, and ontological parsimony can legitimately be invoked to adjudicate between those two rival interpretations. Rather, the point I wish to make in the next sections is that if one takes the statistical interpretation to be preferred because it is a more satisfactory account of population genetics explanations than the causalist interpretation, given that it does not posit any superfluous cause, one finds oneself trapped into the dilemma described above: either one takes statisticalism and 
rejects naturalism; or one rejects statisticalism and stay dissatisfied with the only remaining available account of population genetics explanations, i.e., the causalist account.

\section{Non-Causal Explanations in Science}

Until recently, philosophers thought that scientific explanations need to be causal explanations in order to be genuinely scientific (for a survey, see Woodward, 2017). This is mainly due to the fact that models of scientific explanation which made no reference to causation $^{2}$ were shown to count various non-explanations as explanatory, i.e., they were unable to account for the so-called explanatory asymmetry and avoid irrelevant explanations. Good explanations are indeed thought to be asymmetric. The idea is that if A explains B, $\mathrm{B}$ should not explain $\mathrm{A} .^{3}$ Good explanations need also to be relevant. If $\mathrm{A}$ is completely unrelated to B, even if it is true that whenever A obtains, B obtains, then A is not a good explanation of B. ${ }^{4}$

To face the asymmetry problem and the explanatory irrelevance problem, in the last decades philosophers thought that causal notions should be introduced in our models of scientific explanation to distinguish between regularities that are genuinely explanatory and those that are not (Strevens, 2008). For example, Salmon states that to give "scientific explanations is to show how events and statistical regularities fit into the causal structure of the world" (Salmon, 1977, p. 162). Lewis states that "to explain an event is to provide some information about its causal history" (Lewis, 1986, p. 217). Sober states that the "explanation of an event describes the 'causal structure' in which it is embedded" (Sober, 1984, p. 96).

Philosophers nowadays affirm more rarely that all scientific explanations need to be causal. For instance, Woodward (2003) and Strevens (2008) continue to support the idea that scientific explanations work mainly by describing causal connections, but they both leave open the possibility that there might be scientific non-causal explanations. On the other hand, many philosophers who claim that there are genuine non-causal explanations in science, usually

\footnotetext{
2 The most famous one is the Deductive-Nomological model of scientific explanation (D-N) (Hempel \& Oppenheim, 1948). According to the D-N model, the explanation should take the form of a sound deductive argument in which the explanandum follows as a conclusion from the premises in the explanans, and the explanans must contain at least one law of nature.

3 Several counterexamples to the D-N model were found to show that it was unable to account for explanatory asymmetry. Bromberger's flagpole example is perhaps the most famous one. Cf., e.g., Salmon (1989), p. 47: "A vertical flagpole of a certain height stands on a flat level piece ground. The sun is at a certain elevation and is shining brightly. The flagpole casts a shadow of a certain length. Given the foregoing facts about the height of the flagpole and the position of the sun, along with the law of rectilinear propagation of light, we can deduce the length of the shadow. This deduction may be accepted as a legitimate D-N explanation of the length of the shadow. Similarly, given the foregoing facts about the position of the sun and the length of the shadow, we can invoke the same law to deduce the height of the flagpole. Nevertheless, few people would be willing to concede that the height of the flagpole is explained by the length of its shadow. The reason for this asymmetry seems to lie in the fact that a flagpole of a certain height causes a shadow of a given length, and thereby explains the length of the shadow, whereas the shadow does not cause the flagpole, and consequently cannot explain its height."

4 Several counterexamples to the D-N model were found to show that it was unable to avoid considering irrelevant explanations as genuine explanations. Salmon's birth control pills example is one of the most famous ones. Cf. Salmon (1971), p. 34: "John Jones avoided becoming pregnant during the past year, for he has taken his wife's birth control pills regularly, and every man who regularly takes birth control pills avoids pregnancy."
} 
do not deny that there are also genuine causal explanations in science (see Reutlinger, Saatsi 2018; Lange, 2017). So, despite questions about the metaphysics of causality, such as whether causality finds room in fundamental physics, are still lively debated issues in philosophy of science and metaphysics (for a survey, see Blanchard, 2016), there is still a wide consensus among philosophers of science that causality is relevant to account for explanatory asymmetry and explanatory relevance in scientific explanations.

Whether there are genuine non-causal explanations of particular empirical facts is thus still an unsettled issue (see Reutlinger, Saatsi, 2018 for further references). Several philosophers maintain that there are genuine non-causal explanations in science, while other philosophers either deny that those explanations are genuine explanations, or that those explanations are genuine non-causal explanations (Reutlinger, 2017). For instance, Skow (2014) has argued that existing examples of non-causal explanations are unconvincing, in that they can be accommodated within causal explanation. In order to support his view, Skow weakens the standard causal account of explanation, which requires one to identify the causes of the explanandum, by requiring only that a causal explanation provide some information about the causal history of the explanandum. Skow's notion of 'information' is broader than the notion of 'identifying causes'. So, for instance, according to Skow “one causally explains by merely excluding a possible causal history of the explanandum E, or by stating that $\mathrm{E}$ has no cause at all, while other causal accounts would not classify this sort of information as causally explanatory" (Reutlinger, 2017, p. 4).

For our purposes, it is not relevant to take side on this debate. I just wish to underline that, since statisticalists claim that natural selection is not really a cause of evolution, but they do not deny that the explanations provided by population genetics are genuine explanations, statisticalists are committed to the claim that (at least some) non-causal explanations are genuine scientific explanations, and the explanations provided by populations genetics are explanations of that kind.

The problem is to determine what kind of non-causal explanations population genetics provides. Apparently, several kinds of non-causal explanations can be distinguished in the literature (Reutlinger, 2017). However, as Bokulich states, the literature "on non-causal explanation is still at the stage of trying to find a core set of examples of non-causal explanation that can be agreed upon" and the further task of creating "a taxonomy of the different kinds of non-causal explanation still remain to be done" (Bokulich, 2018, p. 145, fn. 7). It is beyond the scope of this paper to investigate whether those kinds of non-causal explanations can really be distinguished from one another, or whether every kind of non-causal explanations is problematic from a naturalist point of view. As a starting point, it is less contentious and easier to show that at least one kind of non-causal explanations, namely the so-called Mathematical Explanations of Natural Phenomena (MENP), is potentially highly problematic for those who wish to take a naturalist stance. Indeed, MENP are regarded as paradigm example of non-causal explanations in science, and in fact the most promising examples of genuine non-causal explanations in science that one can find in the literature are all explanations where mathematics is thought to play the peculiar role that it is thought to play in MENP. In other words, MENP are the noncausal explanations that better resist the attempts made by the supporters of the causal view on explanation to show that non-causal explanations are either non-explanatory or not really noncausal. So, I will argue, if the statisticalist regards the explanations provided by population genetics as MENP, she has to face the challenge described above. 


\section{Mathematical Explanations of Natural Phenomena}

Briefly, MENP are non-causal, scientific explanations of particular empirical facts in which an indispensable explanatory role is played by a mathematical fact (Baker, 2009). As an example, consider the following question: Why do hive-bee honeycombs have a hexagonal structure? Part of the explanation depends on evolutionary facts. But the explanation is completed by pointing out that the hexagonal tiling is optimal with respect to dividing the plane into equal areas and minimizing the perimeter. This geometrical fact, known as the honeycomb conjecture, was finally proved by Hales in 2001 (Hales, 2001). Thus, the supporters of MENP claim, the explanation of the biological fact that hive-bee honeycombs have a hexagonal structure depends essentially on a mathematical fact (Baker, 2009).

Another famous example of a supposed genuine non-causal explanation is the explanation of the impossibility of taking a walk across the seven bridges that existed in the city of Königsberg at Kant's time over the Pregel river, crossing each bridge only once (Pincock, 2007). The explanation is thought to rest essentially on Euler's theorem, an important theorem in graph theory, according to which a graph can be traversed with each edge traversed only once if and only if it has zero or exactly two vertices with odd degrees (the degree of a vertex of a graph is the number of edges that are incident to the vertex). The graph that describes the Königsberg bridges arrangement has four vertices with odd degrees, thus by Euler's theorem it was impossible to walk across all the seven bridges of Königsberg crossing each bridge only once. Even this example of non-causal explanation is thought to be a MENP, since an indispensable explanatory role is played in it by a mathematical fact, namely Euler's theorem.

Such a dependency on mathematical facts makes MENP both non-causal and nonempirical explanations, since mathematical facts and objects are usually regarded as nonspatiotemporally located and causally inert (Balaguer, 2009), and mathematics is usually regarded as an a priori, i.e., non-empirical, science. For instance, McEvoy writes that on the traditional view of mathematics, mathematical knowledge is "a priori knowledge of necessary truths, and the explanation of why this knowledge is a priori is based on the necessity of these truths: experience alone, that is, cannot tell us what is necessarily true, as opposed to simply true" (McEvoy, 2018, p. 157). ${ }^{5}$

Two clarifications are in order here. First, it is important not to confound alleged instances of MENP with scientific explanations that merely use mathematics for representational purposes. Explanations that merely use mathematics for representational purposes are ubiquitous in science, since science is highly mathematized, but they cannot be regarded as genuine MENP according to MENP supporters. As an example, think of models that represent cardiac electrical activity through a set of coupled nonlinear oscillator equations. We can explain heart functioning as follows. The electrical conduction in the

\footnotetext{
${ }^{5}$ It is worth noting that in the literature on non-causal explanations one can find "different kinds of 'purely' or 'distinctively' mathematical explanations" of particular empirical facts, "such as number-theoretical (Baker, 2009), graph-theoretic (Lange, 2013b; Pincock, 2012), topological (Huneman, 2010; Lange, 2013b), geometric explanations (Lange, 2013b), [...] and statistical explanations (Lange, 2013a; Lipton, 2004)" (Reutlinger, 2017, p. 4). Those kinds of non-causal, mathematical explanations, i.e., MENP, can be distinguished from one another to the extent that the mathematical facts that do the explaining in those explanations belong to different mathematical fields, such as number theory, topology, or graph theory, which can be distinguished from one another. However, since all mathematical fields belong to mathematics, and mathematics can be regarded as a unitary science, those explanations are all MENP and can be treated in a unified manner.
} 
heart allows the propagation of the impulses generated by the sinoatrial node "throughout muscles of atria [...], atrioventricular node [...], and His-Purkinje system [...] to the muscles of ventricles [...]. After the stimulation, the muscles in the walls of atria and ventricles contract providing efficient blood pumping throughout the body" (Ryzhii \& Ryzhii, 2014, p. 67). The similarity between the behaviour of a nonlinear oscillator and the heartbeat "is the motivation to treat the heart as a system of coupled oscillators," where the sinoatrial node as the "primary pacemaker may take the role of the periodic stimulus" (Honerkamp, 1983, p. 69). We can thus simulate the heart electrical behaviour and its possible configurations by using nonlinear oscillator equations. But when it comes to the explanations of the heart behaviour provided by those models, it is what it is represented (e.g., the sinoatrial node activity) that is doing the explaining, not the tool we use to represent it. Indeed, in those scientific explanations where mathematics plays a merely representational role, "the entities' activities themselves are explanatory, not the mathematics that describe them" (Huneman, 2018, p. 119). On the contrary, what happens with MENP is that in those explanations it is mathematics that is "explanatory: mathematical properties are the reason why some facts happen in nature as outcomes or features of the activities" (ibidem, p. 120) of the investigated entities.

Second, MENP are not non-causal explanations in the mere sense that they do not explicitly cite causes. Indeed, there are abstract explanations which do not clearly specify any mechanism or process responsible for the explanandum, but which cannot be regarded as genuine MENP according to MENP supporters (Reutlinger \& Andersen, 2016). As an example, consider models of traffic jam. We can either develop models which mimic the causal effects of the cars on one another, or we can develop models which abstract away from the causal relationships found in the previous kind of models. For instance, we might "imagine a representation of a given system of cars that simply represented what the system would look like after all the cars had achieved some fixed velocity for a given density of cars" (Pincock, 2012, p. 5). In this case, although the explanans abstracts away from specific details of the causal interactions between the components of a system, what is indispensably doing the explaining is not some mathematical fact, as it is supposed to be the case in MENP, rather it is some physical fact (e.g., cars' velocity or density) that can be represented through mathematics in an abstract scenario.

To better understand what kind of explanations MENP are, consider an example provided by Lange (2013b): a mother tries to divide twenty-three strawberries evenly among three children without cutting any strawberry and fails. What explains her failure? According to Lange, the explanation of her failure involves crucially the following assumption: it is a mathematical fact that twenty-three cannot be divided evenly by three. This mathematical fact is both non-causal (since it is a mathematical fact) and explanatory (since it is this very mathematical fact that is allegedly doing the explaining), so that this explanation can be regarded as a genuine MENP. Now, the crucial point is: How it is that a mathematical fact (twenty-three cannot be divided evenly by three) can explain a worldly fact (twentythree strawberries cannot be divided evenly among three children)? According to Lange and other philosophers, mathematical explanations of worldly facts explain in virtue of the extra modal force they have compared to the degree of necessity associated with causal laws, i.e., ordinary laws of nature: that twenty-three is not evenly divisible by three "puts a constraint on any causal laws. There are no causal laws, actual or possible, such that 
they would result in a causal process by which twenty-three strawberries could be evenly divided" (Andersen, 2018, p. 493). According to Glazier, for instance, "it is metaphysically necessary that no one has ever divided 23 objects evenly into thirds, and we may explain why no one has ever done this by saying that it is metaphysically impossible that anyone should have done so" (Glazier, 2017, p. 9). In this view, a MENP provides a so-called metaphysical 'explanation by constraint' of an empirical fact. ${ }^{6}$

Metaphysical explanations by constraint are defined as explanations that explain their targets by showing that they must be the case, since the explanans is more necessary than the explanandum (Bertrand, 2018). MENP are explanations of this kind, since MENP are such that, "given some set of conditions A, then the connection between explanandum and explanans is mathematical in character, such that the resulting explanation involves a modal force stronger than that of causal generalizations" (Andersen, 2018, p. 494). The mathematical fact that twenty-three cannot be divided evenly by three is more necessary than the causal details of mother's particular failure and constrains the way in which any possible attempt to divide worldly objects might be, despite it is a non-causal fact (Bertrand, 2018).

MENP can thus be defined as metaphysical explanations by constrain of particular empirical facts where (a) the explanans is a mathematical fact, and (b) the connection between the explanans and the explanandum is mathematical in character. It has to be stressed that if in MENP the explanans is a mathematical fact, and the connection between the explanans and the explanandum is mathematical in character, since mathematics is usually regarded as necessary, in the sense that mathematical truths are considered to be metaphysically necessary truths, and metaphysical necessity is usually considered to be absolute necessity (see, e.g., Lewis, 1986), so that a mathematical truth is considered to be true in every possible world, and since experience is unable to provide us with such a kind of necessary knowledge (see, e.g., McEvoy, 2018), this means that (a) the explanations provided by MENP are necessary, i.e., they admit of no exception, and (b) in MENP the explanans of an empirical fact is such that cannot be settled or established by experience. Both (a) and (b) set MENP apart from standard scientific explanations, where it is usually thought that it should be possible, at least in principle, to empirically confirm, or disconfirm, the explanans of an empirical explanandum, and where the explanans is usually thought to be contingent, given that even a law of nature is usually thought to be metaphysically contingent, since possible worlds where any given law of nature does not hold are thought to be conceivable, while it is usually assumed that should not be possible to conceive any possible world where a mathematical truth does not hold. Moreover, we have no idea of how it is that we are able to reach such kind of necessary knowledge, while it is widely admitted, even by many non-naturalist metaphysicians and epistemologists, that the challenge to find a reasonable and plausible epistemology for every a priori domain one assumes in one's ontology is a sound and compelling challenge (see, e.g., Peacocke, 1999).

Now, since we are dealing here with scientific explanations, it seems fair to expect that one should be able to provide (1) a scientifically acceptable account of how it is that one can acquire knowledge of the explanans that one is accepting in one's explanation of a given empirical fact, and (2) a scientifically acceptable account of the relation that obtains

\footnotetext{
${ }^{6}$ This is not the only available account of the explanatory power of MENP (see below, fn. 12), but it is certainly one of the most well-developed and widespread, so I stick to it for illustrative purposes. However, many of the concerns raised here about this account can equally be raised about other accounts of the explanatory power of MENP.
} 
between the explanans and the empirical fact to be explained, i.e., an account of how it is that such explanans can explain that fact. When we deal with a standard scientific explanation, we have several possible accounts for how it is that we are able to acquire knowledge of the explanans we are accepting in a given explanation, for instance a physical law, which hinge on the widespread idea that the scientific method allows us to acquire knowledge about the empirical world we live in, albeit fallible knowledge, by observing some inexplicable fact, producing hypotheses to make that fact intelligible, testing those hypotheses, refusing some of those hypotheses, refining some other, and so on. The idea is that a given hypothesis, for instance a given physical law, if it has been empirically confirmed, can explain the fact that it is able to explain because it is an adequate generalization of the domain to which that fact belongs. A given physical law is in a sense homogeneous with the physical fact that it is able to explain, it pertains to the very same empirical domain.

When we come to MENP, things are more complicated, since we do not have any scientifically acceptable account of how it is that we acquire knowledge about mathematics, especially if mathematics, following the standard view in metaphysics and the philosophy of mathematics, is considered to be disconnected from our world and metaphysically necessary. MENP supporters find themselves in the same boat of those platonist philosophers of mathematics who claim at the same time that (a) mathematics exists independently of us and it is causally disconnected from the empirical world, and that (b) we have reliable knowledge about it. In addition to that, MENP supporters claim that mathematics is able to explain empirical facts, not just mathematical facts, because mathematics somehow constrains natural phenomena, but we have no scientifically plausible idea of how it is possible for a mathematical fact to constrain an empirical fact.

\section{MENP and Naturalism}

From what has been said in previous section, it should be easy to see that MENP is very problematic from a naturalist point of view. ${ }^{7}$ Naturalists usually deny that we can have knowledge about non-empirical facts and often are very skeptical about the very notion of metaphysical necessity (Nozick, 2001), so that they usually deny that non-empirical facts can be explanatory. From a broadly naturalist point of view all "knowledge is part of scientific knowledge; natural science is the one and only source of reliable beliefs, including reliable beliefs about the nature of belief itself," and mathematical knowledge "is a part of this" (Brown, 2012, p. 117). In this view, scientific knowledge is acquired using inductive methods, and natural science provides a posteriori knowledge, i.e., knowledge which, in the ultimate analysis, rests on experience (Horsten, 2015). So, also our beliefs about the nature of mathematics and mathematical facts have to be justified in the same way. The main difficulty for MENP supporters is thus to provide a scientifically adequate justification of the claim that mathematical facts constrain natural phenomena.

\footnotetext{
7 In this article the term 'naturalism' is understood as it is usually understood in the philosophy of science and epistemology. In order to avoid any misunderstanding, it is important to note that 'naturalism' is used in a quite different sense in the philosophy of mathematics proper, where it indicates a philosophical position according to which, roughly, the only authoritative standards in the philosophy of mathematics are those of mathematics itself (Maddy, 1997; for a survey, see Paseau, 2013). I will not be concerned in this paper with such philosophical position in the philosophy of mathematics.
} 
I will not be concerned here with any specific view on naturalism, nor I will survey the many criticisms that have been moved to naturalism so far (for a survey, see Papineau, 2016). Indeed, I'm not interested in supporting the claim that, since naturalism is correct, MENP have to be refused. I am merely interested in showing that if one takes a naturalist stance, then one cannot easily accept MENP. For the purpose of this paper, naturalism can be understood as the metaphilosophical stance according to which we should refuse explanations that appeal to non-natural entities, faculties or events, where "non-natural" has to be understood as indicating that those entities, faculties or events cannot in principle be investigated and accounted for in the way we usually do in natural sciences. Although such a characterization of naturalism is quite broad, it nevertheless retains the idea that every naturalist view requires both (a) an ontological and (b) an epistemological commitment. This means that, in order to naturalize a domain $\mathrm{D}$, for instance mathematics, it is insufficient to specify what kind of entities we can admit in our ontology of $\mathrm{D}$, for instance mathematical entities. We have also to provide a naturalistic (i.e., a scientifically plausible and reliable) account of how we can acquire knowledge of those D-entities. ${ }^{8}$ In a very minimal sense, the epistemological commitment of the naturalist can be reduced to the idea that "we should view with suspicion any claim to know facts about a certain domain if we believe it impossible to explain the reliability of our beliefs about that domain" (Field, 1989, p. 233). For this reason, naturalists are usually skeptical about the claim that we can have knowledge of a priori domains, i.e., domains whose objects cannot in principle be empirically investigated (see, e.g., Devitt, 1998).

\footnotetext{
${ }^{8}$ This is the reason why I will not be concerned in this paper with the so-called indispensability arguments for mathematics that have been developed firstly by Quine (1960) and Putnam (1971) in order to defend a realist stance on mathematics (for a survey, see Colyvan, 2019). An indispensability argument is an argument that aims at establishing the truth of some claim by showing the indispensability of the claim in question for a given purpose. In the philosophy of mathematics, an indispensability argument is meant to establish the existence of mathematical entities, provided that they figure in some effective scientific theory. Roughly, the argument goes as follows: if mathematics is indispensable for developing scientific theories, and if one takes scientific theories to be (approximately) true, then one has to conclude that mathematical statements are true too, and so, if one accepts that truth is correspondence, that mathematical entities exist. Now, by relying on an indispensability argument for mathematics, some philosophers, influenced by the Quinean conception of naturalism, ague that if naturalism is interpreted as the claim that philosophy has to be continuous with science and accept in its ontology what scientists accept in their theories, given that scientists seem to accept mathematical entities in their theories, the commitment to the existence of mathematical entities should not be regarded as incompatible with a naturalist stance after all. But the Quinean perspective on naturalism is not the only way to conceive of naturalism and it has so far received several criticisms that have underlined many of its flaws. Some of those criticisms target precisely the claim that we should commit ourselves to the existence of those mathematical entities that figure in our scientific theories. Indeed, that claim leads to an unsatisfactory account of mathematics, since it creates an unpalatable distinction between mathematical entities that can be said to exist because they figure in some scientific theory and mathematical entities that cannot be said to exist because they do not figure in any scientific theory. In this view, pure mathematics has to be regarded as mathematical recreation (Quine, 1960). This distinction is unsatisfactory because very often mathematics is firstly developed as pure mathematics, i.e., without any application in the natural sciences, and only successively find some application. Thus, an account should be provided of "how something that is merely a form of mathematical recreation suddenly raises to the level of serious ontological commitment upon successful application" (Bueno, 2011, p. 364). To avoid those difficulties, here it is assumed that to naturalize a domain D one cannot just defend the claim that the D-entities exist, one has also to provide a scientifically plausible epistemology for the D-entities. The indispensability argument in mathematics is at most able to support the claim that mathematical entities that figure in scientific theories exist, provided that those theories are true, and that truth is correspondence. But it does not provide a scientifically plausible epistemology for the mathematical entities.
} 
So, if in order to explain some fact pertaining a given domain $\mathrm{C}$, one relies on entities that belong to domain $\mathrm{D}$, one needs to provide (1) an account of how it is that one can know D-entities; and (2) an account of how it is that D-entities are able to explain C-facts. Since here we are dealing with the issue of whether MENP are compatible with naturalism, (1) and (2) need to be cast in such a way that can be acceptable from a naturalist point of view.

The difficulty of providing (1), i.e., a naturalist account of how it is that one can know mathematical entities, is due to the fact that mathematics is usually regarded as an a priori domain: mathematical entities are regarded as causally inert and located outside physical spacetime. We currently do not have any scientifically plausible account of how human cognitive faculties could interact with such kind of entities. So, it is difficult to provide an account of how it is that we can have knowledge of the mathematical domain that is continuous with our scientific practice (Field, 1989).

The difficulty of providing (2), i.e., a naturalist account of how it is that mathematical entities are able to explain empirical facts, is twofold. On the one hand, the reason why mathematical facts are able to explain worldly facts is often thought to be by MENP supporters that mathematical facts are modally stronger than physical facts (Lange, 2013b), or that mathematical facts provide modal information about empirical facts (Berenstain, 2017). But if one provides this kind of reasons, one just begs the question. In order to explain the relationship between mathematical facts and worldly facts, one resorts to entities or facts which belong to another domain, namely the modal domain. Now one needs to provide an account of how it is that one can have knowledge of modal facts. The problem is that the modal domain is usually regarded as an a priori domain in its turn, and modal facts are thought to be non-empirical in the same sense in which mathematical facts are thought to be non-empirical facts (Jonas, 2017). So, it is not easy to provide a naturalist account of how it is that we can have knowledge of the modal domain either. ${ }^{9}$

On the other hand, there is the difficulty of justifying the claim that there is a sort of connection between the mathematical domain and the empirical domain, and that this connection is mathematical in character. As Mancosu states, how "the connection between mathematics and the world is to be accounted for remains one of the most challenging problems in philosophy of science, philosophy of mathematics, and general philosophy" (Mancosu, 2018, Sect. 1). For example, Lyon (2014) discusses the usual textbook answer to the question: Why are normal distributions normal? Normal distributions (i.e., Gaussian distributions or bell curves) are ubiquitous in science, and the explanation usually provided for why they are normal is that they are normal because of the Central Limit Theorem (CLT) from probability theory. Briefly, the CLT says that if a random variable $X$ is the sum of a large number of small and independent random variables, then independently of how the small random variables are distributed, $\mathrm{X}$ will be approximately normally distributed. Common textbooks explanations refer to the CLT in order to explain why "in a large number of important applications, we meet distributions which are at least approximately normal," such in the case, for instance, of "a great number of demographical and biological distributions [...]. The central limit theorem affords a theoretical

\footnotetext{
9 The difficulty of providing a naturalist account for mathematics and modality derives from the fact that both mathematics and modality have to face the access problem. The access problem, first raised in the philosophy of mathematics by Benacerraf (1973), is now thought to arise in many other domains (see, e.g., Jonas, 2017). It is the problem of justifying the claim that our D-beliefs align with the D-truths of a given domain $\mathrm{D}$, if $\mathrm{D}$ is regarded as an a priori domain, i.e., a domain whose objects cannot in principle be empirically investigated. Both mathematics and modality are usually regarded as a priori domains.
} 
explanation of these empirical facts" (Cramer, 1946, p. 231). This is clearly an example of MENP and might appear to be a satisfactory scientific explanation. But Lyon underlines precisely the problem we are dealing with. Indeed, taken at face value, such an explanation cannot be true, nor satisfactory, since it "surely can't be the theorem by itself that is doing the explanatory work," given that at "the very least, there must be additional premises that connect the theorem to their explananda. Without additional premises, the theorem is explanatorily idle, disconnected from any empirical phenomena" (Lyon, 2014, p. 626). So, in order for such an explanation to be a satisfactory explanation, we should make explicit the premises that "connect the theorem to quantities and give the explanations in full detail," but this might turn out to be "surprisingly difficult-so difficult that it seems that the CLT does not explain why quantities are normally distributed as often as the textbooks suggest" (ibidem, p. 627).

To understand why it might be so difficult to connect the theorem that is supposed to do the explaining to the empirical explanandum, one has to keep in mind that when one deals with MENP, the relation that connects the theorem with the explanandum cannot be one of 'representation', 'analogy', or 'approximation', as it is the case in standard scientific explanations that merely use mathematics for representational purposes, as for instance in the example made above of the mathematical models of cardiac electrical activity, since, as already noted, in genuine MENP mathematics plays no representational role. But if one excludes the representational relation between mathematics and the empirical fact that a mathematized explanation aims to explain, few options to construe the connection between the mathematical explanans and the empirical explanandum are left, and none seems to be compatible with a naturalist stance. The problem is that it is not easy to envisage how a naturalistic epistemology might be able to justify the claims that MENP supporters make about mathematics and its relation to empirical facts (Bueno, 2017). Indeed, if one assumes, for instance, that mathematical facts are able explain empirical facts because mathematical facts are able to somehow transmit their stronger modal force to natural facts, one has to provide a scientifically satisfactory account of how this transmission occurs. As Baron writes, whenever one is confronted with an alleged case of MENP, a case in which the mathematics is constraining the physical world "in some modally robust way, a natural question arises as to how it is that mathematics - something that appears to be metaphysically disconnected from the physical world - might place such incredible constraints on physical systems" (Baron, 2014, p. 472). ${ }^{10}$

\footnotetext{
${ }^{10}$ If one claims, for instance, that mathematics dictates to us how thing must go in the physical domain because the mathematical domain and the physical domain are isomorphic, then one's view risks becoming a sort of Pythagoreanism, which is certainly a legitimate philosophical view, but which is usually regarded as incompatible with a naturalist stance (Steiner, 1998). Indeed, an isomorphism can obtain only between two mathematical structures, so if one claims that the physical domain is isomorphic to the mathematical domain, one has to conclude that the physical universe is a mathematical structure. Tegmark, for example, explains the utility of mathematics for describing the physical world "as a natural consequence of the fact that the latter is a mathematical structure, and we are simply uncovering this bit by bit. [...]. In other words, our successful theories are not mathematics approximating physics, but mathematics approximating mathematics" (Tegmark, 2008, p. 107). Indeed, from the definition of a mathematical structure, it follows that "if there is an isomorphism between a mathematical structure and another structure [...], then they are one and the same. If our external physical reality is isomorphic to a mathematical structure, it therefore fits the definition of being a mathematical structure" (ibidem). So, if we accept the idea that mathematics directly explains worldly facts, because mathematics sets the boundaries of what can happen in the physical universe, we risk becoming unable to distinguish the mathematical from the physical. This is a variant of what French (2014) calls the "Collapse Problem" for structural realism. French's proposed solution to avoid the Collapse Problem and distinguish the physical from the mathematical is to take into account causation. Obviously, since here we are dealing with MENP, which, by definition, are non-causal explanations, French's proposal cannot be of use.
} 


\section{Three Objections and Replies}

Three main objections can be raised against the claim I made in the previous section that naturalism is incompatible with MENP.

First Objection. The explanation provided of why it is difficult to combine naturalism with MENP implicitly assumes a causal view of scientific explanation, but whether a causal view of explanation is legitimate, or whether it should be regarded as the only legitimate one, it is precisely what is at stake, so it is unfair to claim that MENP are not compatible with a naturalist stance by assuming such a view on scientific explanation.

Reply. Assuming a causal view on explanation is not necessary in order to point out the difficulty of giving a naturalist account of our knowledge of the mathematical and the modal domains. Even if we assume that non-causal explanations are legitimate scientific explanations, the problem is still with us, since the point is that there is no adequate account of how it is that we can have knowledge of mathematical entities or modal facts which is compatible with our current scientific knowledge. And this fact is independent from whether such knowledge is cast in causal or non-causal terms. Consider mathematics. The problem of reconciling our philosophical reflections on the nature of mathematics with a scientific worldview is far from being solved, and despite mathematics is so indispensable for scientific knowledge, we do not have yet an uncontroversial and science-oriented account of what mathematics is and how we can acquire mathematical knowledge. The problem of providing a satisfactory naturalist account of mathematics is so far from being solved that Leng states that "the most obvious answers to the two questions 'What is a human?' and 'What is mathematics?' together seem to conspire to make human mathematical knowledge impossible" (Leng, 2007, p. 1). So, the problem of providing an adequate scientific account of mathematical knowledge is still pressing for those who claim that mathematics is able to explain empirical facts by itself even if one regards non-causal explanations as acceptable. Since the way in which I described the difficulties of making MENP compatible with naturalism does not indispensably rest on the assumption of a causal view on scientific explanation, this objection is inadequate.

Second Objection. The explanation provided of why it is difficult to combine naturalism with MENP implicitly assumes a causal theory of knowledge, according to which an epistemic subject can have knowledge of a given entity in virtue of a causal relation that obtains between the epistemic subject and such entity. But the causal theory of knowledge is now refused by many philosophers, and, again, that causation has to be regarded as indispensable for a naturalist perspective to be defined as such is something that should be argued for and not just assumed.

Reply. This objection is related to the previous one and resembles an objection that has been moved against Benacerraf's (1973) criticism of mathematical Platonism, which relied on the causal theory of knowledge, a theory which nowadays is discredited among epistemologists. But Benacerraf's argument may be raised against mathematical Platonism without assuming the causal theory of knowledge, as Field (1989) pointed out first. As Baron clearly summarizes:

The mathematical propositions that mathematicians believe tend to be true. If platonism is correct, however, then these propositions are about mathematical objects. So, the mathematical beliefs held by mathematicians $[. .$.$] are reliably correlated with$ facts about such objects. The challenge facing the platonist, then, is to provide an account of this reliable correlation. (Baron, 2015, p. 152). 
As already noted, the problem is how to provide an account of how it is that our D-beliefs align with D-truths, if domain D is an a priori domain. Even if we accept noncausal explanations, the 'reliable correlations' between our D-beliefs and D-truths must be explained in a way that is compatible with our current scientific understanding of the world. Consider how many mathematical Platonists account for how it is that we can know mathematical entities. Many of them claim that there is a sort of intellectual faculty that we are supposed to possess, a faculty that would allow us to "see", for instance, that a given set of axioms is consistent, even if we cannot formally prove that it is so because of Gödel's results. For example, Gödel himself, who embraced Platonism, states that "despite their remoteness from sense experience we do have something like a perception also of the objects of set theory, as is seen from the fact that the axioms force themselves upon us as being true" (Gödel, 1990, p. 268), even if we cannot formally prove their consistency. In the same vein, Brown states that we "can intuit mathematical objects and grasp mathematical truths. Mathematical entities can be 'perceived' or 'grasped' with the mind's eye" (Brown, 2012, p. 45). But no-one of such Platonist philosophers of mathematics is able to provide a scientifically plausible account of such hypothesized intellectual faculty, nor is anyone able to even prove that it actually exists. For instance, Brown admits that he has "no idea how the mind is able to 'grasp' or 'perceive' mathematical objects and mathematical facts" (ibidem, p. 12). According to Gödel, there "exists [...] an entire world consisting of the totality of mathematical truths, which is accessible to us only through our intelligence," a world which was "divinely created" (Gödel, 1990, p. 323). Gödel also explicitly claims that our mathematical intuition is a superior faculty which is not "derived from [...] Darwinian adaptation" (Gödel, 1995, p. 354). Such accounts are clearly inadequate from a scientific perspective, and so are unacceptable to naturalists. A naturalist account of mathematics has to assume the hypothesis that the human mathematician is "a thoroughly natural being situated in the physical universe," and that therefore "any faculty that the knower has and can invoke in pursuit of knowledge must involve only natural processes amenable to ordinary scientific scrutiny" (Shapiro, 1997, p. 110). So, the issue is not that a causal explanation of how such intellectual faculty might work should be given, rather the issue is that at least a scientifically plausible account of how such faculty works should be given, be it causal or non-causal. The problem is that, as already noted, no scientific account of how such faculty works is usually provided, not even a non-causal one. Since the way in which I described the difficulties of making MENP compatible with naturalism does not indispensably rest on the assumption of a causal theory of knowledge, this objection is inadequate.

Third Objection. The explanation provided of why it is difficult to combine naturalism with MENP assumes a realist perspective on both mathematical and modal facts, and certainly it can be hard to accommodate such realist perspective within a naturalist framework, but that view on mathematics and modality is not the only legitimate one, and other views could be made compatible with a naturalist stance more easily, so it is unfair to claim that MENP are incompatible with naturalism by assuming such a realist perspective on both mathematical and modal facts.

Reply. This objection requires to be carefully handled, since it seems prima facie a very reasonable and compelling objection. But when analysed more closely, it is not so. The point is the following: true, there are different non-realist ways to conceive of mathematical and modal facts; true, those non-realist views are philosophically respectable views; and true, (at least) some of those views could more easily be made compatible with naturalism; but if one adopts some of those non-realist perspectives on mathematical and modal facts, one risks being unable to claim (and account for how it is) that mathematical and modal facts can explain empirical facts, as MENP supporters wish instead to claim. 
Indeed, usually those who claim that MENP are genuine scientific explanations take a realist view on mathematics and modality precisely because they wish to maintain that some empirical fact can be scientifically explained by some non-empirical fact. Briefly, scientific explanations are usually regarded as objective because they are regarded as mind-independent, i.e., independent from the epistemic subject. This means that both the explanandum and the explanans need to be mind-independent. In the case of MENP, the explanans is a mathematical fact, so if MENP have to be regarded as genuine scientific explanations, mathematical facts should be regarded as mind-independent facts in the same sense in which a physical fact is regarded as mind-independent in ordinary scientific explanations. Now, the idea that mathematical facts are mind-independent is usually associated with a realist stance on mathematics. So, usually MENP supporters do not advocate for any anti-realist perspective on mathematics.

An analogous reasoning can be deployed in the case of modality. True, modal realism is not the only possible view on modality. Nevertheless, it is also easy to understand why modal realism is so appealing to MENP supporters. Indeed, if one takes an anti-realist stance on modality, usually one refuses the claim that modal facts are mind-independent facts in the same way in which physical facts are thought to be mind-independent in ordinary scientific explanations. For instance, van Fraassen, who embraces an anti-realist view on modality, states that the "modal discourse describes features of our models, not features of the world" (van Fraassen, 1989, p. 214). On the contrary, according to modal realists, modal truths are made true by the same kind of correspondence with reality that makes empirical claims true. Thus, the main motivation for realism about modality "comes from a commitment to the mind-independence of the truth-makers for modal claims. The core idea is that what makes a possibility or necessity claim true is not some fact about human minds, but some fact about the entities themselves" (Vaidya, 2016, Sect. 1.2.3).

Different ways to conceive of modal realism are available in the literature. But the point here is not which formulation of modal realism one adopts. Rather the point is whether one can provide a justification for the claim that we can have knowledge of metaphysical necessities that is acceptable from a naturalist point of view, if one adopts a realist view on modality. It is indeed difficult to combine the claim that we can have knowledge of what is necessary by reasoning alone with the claim that reasoning is a natural faculty, which is limited and fallible since it evolved through natural selection. Again, the difficult issue is to provide a scientifically plausible justification of the metaphysically bold claim that we are extremely reliable when we deal with a priori domains.

How can we have reliable knowledge of what is necessary? Most philosophers agree that from what is actual we can make some safe inferences on some kind of possibilities. The difficult issue is how to justify claims on what is necessary, i.e., what is true in all possible worlds. The difficulty is that there is not a fully developed naturalist epistemology well suited for modal realism already available (see Nolan, 2017, for a plea to develop a naturalist approach to modal realism). Indeed, if one investigates on what, in the ultimate analysis, philosophers or mathematicians rely in order to claim that some proposition expresses a metaphysically necessary truth $\mathrm{T}$, one will find some intuition that it cannot be the case that $\mathrm{T}$ does not hold. Now, if this intuition is due to an evolved and fallible human cognitive faculty, then it is insufficient to justify the claim that we are reliable when dealing with metaphysical necessity, since the domain of what is metaphysically necessary extends far beyond those contexts that is reasonable to think that our ancestors needed to address in order to survive (Nozick, 2001). If, on the contrary, the reliability of our intuition in dealing with metaphysical necessity is guaranteed by some non-natural fact or faculty, then we have to dismiss naturalism. 
Contrary to the claim that there is not a naturalist and realist account of modality, it might be objected that a counterfactual theory of modal knowledge may be able to pave the way for the development of a naturalist perspective on modality. Williamson (2007) famously develops an analysis of modal knowledge based on counterfactual reasoning, moving from the works of Stalnaker (1968) and Lewis (1973). According to Williamson, the epistemology of metaphysical modality must somehow derive from our ordinary cognitive capacities. In this view, it is our ability in performing counterfactual reasoning that justifies our reliability about modality. Williamson shows that statements about metaphysical possibility and necessity can be proven to be logically equivalent to counterfactual conditionals (Vaidya, 2016, Sect. 3.1). According to Williamson, "the epistemology of metaphysically modal thinking is tantamount to a special case of the epistemology of counterfactual thinking" (Williamson, 2007, p. 158). Since our ability to perform counterfactual reasoning might be regarded as very useful for the survival of our ancestors, it might be the case that such ability has been selected for. So, despite Williamson aversion to naturalism, Williamson's view may represent a way to naturalistically justify our reliability about modality.

The truth conditions for subjunctive conditionals are the following: a subjunctive conditional $\mathrm{A}>\mathrm{C}$ (if it were the case that $\mathrm{A}$, it would be the case that $\mathrm{C}$ ) is true at a possible world $w$ just in case either (1) A is true at no possible world or (2) some possible world at which both $\mathrm{A}$ and $\mathrm{C}$ are true is more similar to $w$ than any possible world at which both $\mathrm{A}$ and $\neg \mathrm{C}$ are true. Williamson's basic epistemic idea is that a justified belief about necessity can be arrived at through a counterfactual development, in imagination, of the supposition that $\neg \mathrm{A}$ (Vaidya, 2016). In this view, it is necessary that A if and only if were $\neg \mathrm{A}$ true, a contradiction would follow.

Now, is Williamson's account of modal knowledge really an adequate naturalist account when one comes to metaphysical necessity? The answer seems to be in the negative. Let us briefly see why. Williamson's method for counterfactual reasoning can be summarized as follows: one supposes the antecedent of a counterfactual and develops the supposition, adding further judgments within the supposition by reasoning and background knowledge. One asserts the counterfactual if and only if the development of the antecedent eventually leads one to add the consequent (Williamson, 2007). The crucial point is that what constraints one's imagination when one develops scenarios searching for whether a contradiction would follow is one's "background knowledge." Williamson claims that our imagination is somehow restricted by our "sense" of how nature works. Background knowledge can be regarded as deriving from experience or can be regarded as instilled in us by natural selection. Now, if on the one hand, the reliance on background knowledge makes Williamson's proposal compatible with a naturalist stance, on the other hand such reliance on background knowledge constitutes an insurmountable difficulty for Williamson's view. Indeed, as Tahko (2012) pointed out, if our background knowledge concerns the laws of nature familiar from the actual world, how can we reliably restrict our imagination regarding scenarios that do not concern the actual world? If we wish to account for metaphysical necessity, i.e., for what is necessary in all possible worlds, we need to be reliable also with respect to scenarios far removed from the actual world. Indeed, in order to develop any given scenario when performing counterfactual reasoning, one has to be reliable in the choice of what should be held fixed and what can be modified in the antecedent. Since laws of actual world could be metaphysically contingent, if it is our background knowledge that dictates to us what facts are metaphysically necessary and hence should be held fixed, this "leaves entirely open how we are supposed to know which items of our background knowledge are metaphysically necessary" and hence should be regarded as "constitutive facts" 
and held fixed in every possible scenario (Tahko, 2012, p. 105). Our epistemic access to these modalities cannot be in terms of counterfactuals, otherwise Williamson's account would be circular, "as constitutive facts are supposed to help us to get the account started in the first place" (ibidem).

So, Williamson's method for counterfactual reasoning can only tell us that if a certain fact is metaphysically necessary, then varying it in a counterfactual antecedent will generate a contradiction, but it seems unable to tell us what facts are metaphysically necessary. To correctly perform counterfactual reasoning, one must have some modal knowledge of metaphysical necessities that precedes the counterfactual assessment. In other words, Williamson's method might be reliable if we happened "to hold fixed the correct facts, but we have no means to determine whether this is indeed the case. [...]. We would need prior access to metaphysical necessities to enable this, but that is exactly what Williamson denies" (ibidem, p. 108). Since it is not able to identify what facts are metaphysically necessary, Williamson's counterfactual theory of modal knowledge does not really provide us with a naturalist and realist epistemology of modality. From what we said, it seems fair to suspect that analogous difficulties may potentially arise with every other attempt which aims at developing a naturalist and realist epistemology of modality by assuming a counterfactual theory of modal knowledge.

Since the way in which I described the difficulties of making MENP compatible with naturalism does not indispensably rest on the assumption that MENP supporters need to endorse mathematical realism or modal realism, but on the more modest assumption that usually MENP supporters endorse some realist stance on mathematics and modality because there is not an easy way to develop an anti-realist stance on mathematics and modality suitable to be combined with the claims made by MENP supporters about how it is that MENP are explanatory, this objection is inadequate.

\section{Statistical Explanations in Population Genetics}

Turning to the issue at stake, the point is that if one adopts statisticalism and accepts the claim that the explanations provided by population genetics are genuine MENP, then one cannot easily proclaim oneself a naturalist. Indeed, MENP seems to be incompatible with a naturalist stance, since there is no available naturalist account of how MENP work. Consider that it is undisputed that statisticalists are "committed to a class of statistical, noncausal explanations" (Walsh et al., 2017, p. 6). So, to determine whether or not statisticalists need to address the present challenge, the only point still in question is whether the explanations in that class of statistical, non-causal explanations can be regarded as genuine MENP.

If this is the case, the statisticalist faces a dilemma: either she rejects naturalism, an option that might be unpalatable to many statisticalists who see themselves as naturalists; or she rejects the claim that there are genuine MENP, but this would amount to reject statisticalism and accept the causalist view. To escape this dilemma, the statisticalist should be able to prove (1) that the statistical explanations provided by population genetics are not genuine MENP, but (2) can nevertheless be regarded as non-causal explanations, and (3) that those statistical, non-causal explanations are compatible with a naturalist stance, i.e., it is possible to provide a naturalist account of how those non-causal explanations work. In the rest of the paper, I aim to show that it is not easy for the statisticalist to prove (1), (2), and (3). 
The first question to address is: If one adopts statisticalism, is one forced to accept the claim that all the statistical explanations provided by population genetics are genuine MENP? It might be difficult to answer that question, since it might be difficult to prove that all the statistical explanations provided by population genetics are genuine MENP. ${ }^{11}$ How could one prove such claim once and for all? Let us put aside the indirect argument that several philosophers used statistical explanations from population genetics as examples of genuine MENP. This might be irrelevant, since those philosophers might have been mistaken. Moreover, even if those explanations were genuine MENP, this does not exclude the possibility that there might be other statistical explanations in population genetics that are both non-causal and non-mathematical. However, this formulation of the issue is perhaps unnecessarily strong. For the present challenge to be taken seriously by statisticalists, it suffices that at least some statistical explanations in population genetics are genuine MENP, it is not necessary that all statistical explanations in population genetics are MENP. This is why it is better to say that the statisticalist has to prove (1'), i.e., that statistical explanations from population genetics that are usually regarded as genuine examples of MENP are not really MENP. In other words, it is insufficient for the statisticalists to show that there are some statistical explanations in population genetics that are not genuine MENP in order to claim that the present challenge need not be addressed. To make such claim, statisticalists would have to show that there are no MENP at all among population genetics explanations. Indeed, if at least some explanations in population genetics are MENP, the statisticalist should need to address the present challenge with regard to those cases. If the statisticalist is able to prove $\left(1^{\prime}\right)$, she then has still to show (2), i.e., that the statistical explanations in population genetics that are not MENP are genuinely non-causal (otherwise her position would be pointless), and (3), i.e., that that kind of non-causal explanations is compatible with a naturalist stance.

Let us focus on the difficulty of proving (1'), i.e., that statistical explanations from population genetics that are usually regarded as genuine examples of MENP are not really MENP. The problem is that some of the alleged examples of MENP which come from population genetics are quite convincing.

Consider, for instance, the case of drift discussed above in Sect. 2. Drift explanations are regarded by statisticalists as genuine examples of statistical, non-causal explanations in science (Walsh et al., 2017). Are drift explanations genuine MENP? The answer to this question seems to be in the affirmative. Lange (2013a), for instance, conceives of drift explanations as genuine MENP. According to Lange, drift explanations in population genetics are what he calls Really Statistical (RS) explanations. In Lange's view, an RS explanation "does not proceed from the particular chances of various results [...]. It exploits merely the fact that some process is chancy" (Lange, 2013a, p. 173). RS explanations are statistical explanations that are thought to be genuine instances of MENP, since it can be shown that RS explanations are both (a) genuine non-causal explanations, i.e., they are not explanations that merely fail to cite causes; and (b) genuine mathematical explanations, i.e., they are not explanations that merely use mathematics for representational purposes.

\footnotetext{
11 Otsuka is less cautious on this issue and straightforwardly affirms that, according to the statisticalists, "population genetics is not only mathematical-it is a mathematics. That is, not only does it deal with complex mathematical formulae (after all physics is full of sophisticated mathematics), but rather its principal equations describing evolutionary changes are all mathematical theorems, whose derivation requires nothing more than assumptions or axioms of, say, probability theory" (Otsuka, 2016, p. 461).
} 
As regard (a), in order to illustrate in what sense RS explanations are non-causal explanations, Lange considers explanations which work by identifying the result being explained as an instance of some characteristically statistical fact, such as regression to the mean, departure from the expectation value, and random walks. According to Lange, all statistical explanations that explain a given empirical fact $\mathrm{X}$ by appealing to one of those statistical facts are genuine MENP, since those statistical facts rest on some theorem from probability theory, i.e., on mathematics. Drift explanations explain why frequencies depart from their expectation values and why these departures tend to be greater for smaller populations. As already noted, a crucial role is indeed played by population size in drift explanations. Despite some philosophers tried to provide a causal construal of the role played by population size in drift explanations, given that population size can be manipulated, according to Lange drift explanations are instead non-causal explanations, since in drift explanations "the explanatory power of effective population size" is "mediated not by any laws of nature, but merely by the 'laws of probability' - that is, by math. No causal law is responsible for making greater departures from expectation values more likely as population size diminishes" (ibidem, p. 184).

As regard (b), in order to explain why RS are genuine mathematical explanations, Lange points out that RS explanations do not appeal to any particular aspects of what needs to be explained, "but merely to the fact that there are chances. Consequently, instead of subsuming the result to be explained under a statistical law of nature, an RS explanation exploits a theorem of the probability calculus" (ibidem, p. 177). This point is crucial, since insofar as "mathematical facts alone are emphasized as doing the explaining," an explanation "is properly characterized as distinctively mathematical" (Lange, 2013b, p. 507), i.e., as a MENP. So, if in every RS explanation it is some theorem of probability theory that is mainly doing the explaining, since probability theory is a mathematical field, then RS explanations are genuine MENP, i.e., RS explanations are both non-causal and mathematical. Thus, if drift explanations are RS explanations, drift explanations are genuine MENP. ${ }^{12}$

Lange's line of reasoning seems (at least prima facie) sound and so worth being taken seriously. And so are other examples of MENP that come from population genetics provided by MENP supporters. Thus, if a statisticalist wishes to deny, for instance, that drift explanations in population genetics are RS explanations, and so that can be regarded as genuine MENP, she has to provide reason for either (a) the claim that drift explanations are not really non-causal, or (b) the claim that in drift explanations it is not some mathematical theorem that is mainly doing the explaining.

Both options are problematic for the statisticalist. As regard (a), it is easy to see that a statisticalist cannot take it, since statisticalists claim precisely that drift explanations in population genetics are non-causal explanations. As regard (b), also this option appears to be difficult to take for the statisticalist, since it seems that statisticalist $d o$ claim that in statistical explanations theorems from probability theory play an indispensable explanatory role. Evidence for this claim comes from the very way in which statisticalists themselves

\footnotetext{
12 To be precise, Lange distinguishes between MENP that are explanations by constrain (as in the case of mother and twenty-three strawberries) and MENP that are RS. Indeed, if both of those kinds of MENP explain by showing that "the fact to be explained could not have been otherwise" (Lange, 2017, p. 5), RS explanations do not "appeal solely to "constraints' such as mathematical necessities," but also to the "contingent fact [...] that a given system is statistical" (ibidem, p. 196). I do not consider this distinction here, since it does not impinge on the main aim of the paper.
} 
describe in their works the statistical, non-causal explanations which, in their view, characterize population genetics.

Recall that in order to deny that statistical explanations in population genetics are genuine MENP, the statisticalist should be able to show that those statistical explanations are both non-causal and non-mathematical. This prima facie might appear to be a viable possibility, but in fact things are more complicated. Indeed, it is certainly true that many authors think that there can be both statistical explanations that are causal and statistical explanations that are non-causal. The problem is that when it comes to non-causal, statistical explanations in population genetics, the examples provided are always MENP, i.e., noncausal, mathematical explanations.

For instance, Lange (2017) claims that in many cases there can be two statistical explanations of the very same empirical phenomenon, one that is causal and the other one that is non-causal. As an example, he considers the case of a fair coin that is tossed 100,000 times. If we consider various runs of 20 consecutive tosses beginning with toss numbers $1,11,21$, etc., we have that neighboring runs share 10 tosses, and so that a run with more than 10 heads tends to be followed by another run with more than 10 heads. Nevertheless, a run with an exceptionally high number of heads tends to be followed by a run with fewer heads. According to Lange, there are two statistical explanations for this result. In the first explanation, this result is explained by regression toward the mean. In the second explanation, this result is explained by the coin's $50 \%$ chance of landing heads on any given toss (independently of the outcomes of other tosses): "if we compute the chance that a run with an exceptionally high number of heads will be followed by a run with fewer heads, we will find the chance to be high" (Lange, 2017, p. 191). In Lange's view, both of these "explanations are aptly termed 'statistical'. However, they are fundamentally dissimilar. The explanation that does not appeal to regression toward the mean is causal whereas the regression explanation is non-causal" (ibidem).

The statistical explanation that does not appeal to regression toward the mean is causal because it appeals "to the coin's fairness - that is, to the propensity of the coin (together with the coin-tossing mechanism and background conditions) to produce a 'heads' outcome," and this propensity is a disposition. Now, even if "whether or not a disposition is a cause of its manifestation, an explanation of some result that appeals to the underlying disposition manifested in the result is 'causal' in the broad sense" that the explanation derives its explanatory power from "describing relevant features of the result's causal history or, more broadly, of the world's network of causal relations" (ibidem).

On the contrary, the statistical explanation that appeals to regression toward the mean is non-causal, since in this explanation it is a theorem from probability calculus that is mainly doing the explaining, and the explanation does not derive its explanatory power from its describing relevant features of the result's causal history. In Lange's view, this explanation is an RS explanation, and so it is a MENP.

It is worth underlying that Lange's broad view on causality is in line with the broad view on causality defended by those philosophers who tried to show that alleged examples of non-causal explanations are not really non-causal, such as Skow (2014). In other words, this broad view on causality is one of the most permissive account of causality available in the literature, and so it is also one of the most restrictive criteria for non-causality. As a result, in Lange's view the only genuine non-causal explanations are MENP. Indeed, MENP are non-causal "because they do not work by supplying information about a given event's causal history or, more broadly, about the world's network of causal relations," rather MENP work "by showing how the fact to be explained could not have been otherwise" (Lange, 2017, p. 5), given that in MENP the explanans is mathematical in character. Other philosophers 
share Lange's view (see, e.g., Reutlinger, 2017), so the point is that it seems fair to say that many philosophers nowadays think that if a statistical explanation is non-causal, then it is a MENP. Obviously, there is not a rigorous demonstration that tertium non datur. However, there are plausible reasons to think that the possibility of identifying uncontroversial instances of non-causal and non-mathematical statistical explanations is excluded by the fact that the supporters of the causal view on scientific explanation, in order to deny that there are non-causal explanations in science, broadened their notion of causality to the extent that the only non-causal explanations that cannot be accommodated by their view on causality are MENP, given that mathematics is almost universally regarded as non-causal. This means that if one claims that a given statistical explanation is non-mathematical, then one risks being unable to defend the claim that that statistical explanation is also non-causal.

The big problem for the statisticalists is that they seem to agree with those philosophers who think that either a statistical explanation is causal, or it is a MENP. Indeed, the way in which statisticalists themselves describe the statistical, non-causal explanations one can find in population genetics seems to perfectly fit the definition of MENP given above (Sect. 4). More precisely, those explanations seem to perfectly fit the definition of RS explanations given by Lange (2013a), which, as noted above, are genuine MENP. For instance, Ariew et al. (2015) claim that all the explanations provided by "the modern genetical theory of natural selection are 'autonomous-statistical explanations' analogous to Galton's explanation of reversion to mediocrity" (Ariew et al., 2015, p. 635). Galton was interested in explaining the stability of variation for quantitative features of populations, such as height, from generation to generation. Galton showed that the differences in the quantitative characters "conforms to a single statistical law, the 'law of deviation' which Galton demonstrated could be graphically represented by a bell-shaped normal distribution" (ibidem, p. 644). Galton showed that the normal distribution regenerate itself at each generation: in a second generation there will be a normal distribution of the same mean and variance. In his explanation Galton does not make reference to any causal factor, he just appeals to "the deductive consequences of laws that involve the statistical properties of the population, namely, the mean and variance of the normal distribution" (ibidem, p. 644). In Galton's explanation, the normal distribution regenerates itself at each generation because of regression toward the mean, and so, in the ultimate analysis, it is a theorem from probability theory, namely the CLT discussed above, that is mainly doing the explaining in Galton's statistical explanation. This might let us think that Galton's explanation is conceived as a genuine MENP by Ariew et al. (2015). This hypothesis is confirmed by Ariew, Rice, and Rohwer themselves. In a related paper (Ariew et al., 2017) the Authors explicitly claim that "Galton's explanation [...] is surprising because his explanation only makes reference to a mathematical result" (Ariew et al., 2017, p. 64), i.e., in Galton's explanation the explanans is a mathematical fact. They also claim that in explanations such as that provided by Galton, "the statistical laws do all the explaining without the need to refer to any causal features of the ensemble" (ibidem). Moreover, they claim that in Galton's explanation the fact to be explained is "a mathematical consequence of the law of deviation" (ibidem, p. 70), i.e., in Galton's explanation the connection between the explanans and the explanandum is mathematical in character. This description of Galton's explanation seems to perfectly fit MENP's definition. ${ }^{13}$

\footnotetext{
13 To be precise, Ariew et al. (2017) claim that Galton's explanation is not an RS explanation as defined by Lange (2013a), and that their account of 'autonomous-statistical explanations' has to be preferred to Lange's one. Nevertheless, they do not reject Lange's view on statistical explanations because they wish to deny that statistical, non-causal explanations are MENP. Rather, they criticize Lange's claim that it is pos-
} 
In addition to that, in Ariew's, Rice's, and Rohwer's account, it seems that Galton's explanation can be regarded as non-causal for the very reason that it is mathematical. They write that "Galton did not interpret the statistical distribution as favouring a non-probabilistic causal hypothesis" (Ariew et al., 2015, p. 644). This seems to mean that in their view the bell-shaped normal distribution discussed by Galton could be explained either by a causal and non-probabilistic hypothesis or by a non-causal and probabilistic hypothesis. Since probability theory is a mathematical field, we can fairly read "non-causal and probabilistic" as equivalent to "non-causal and mathematical." It seems thus fair to conclude that according to Ariew, Rice, and Rohwer, statistical explanations can be either causal or distinctively mathematical, i.e., MENP.

Now, since Galton's explanation is an explanation by regression toward the mean, for all it has been said, it seems that it can safely be regarded as a MENP. Lange himself makes reference to Galton's result in discussing his definition of RS explanation: since "the explanation of the coin-toss result by regression toward the mean is an RS explanation rather than a causal explanation, it unifies the coin-toss result with [...] Galton's result regarding children's heights" (Lange, 2017, p. 193). This example suggests that statisticalists think that all the statistical and non-causal explanations one can find in population genetics are genuine MENP, and that there is no room for statistical explanations in population genetics that are both non-causal and non-mathematical.

Footnote 13 (continued)

sible to distinguish a statistical, causal explanation from a statistical, non-causal explanation of the same empirical fact, such as in the example of the various runs of 20 consecutive coin tosses described above which, according to Lange, might be explained either by regression toward the mean or by calculating the probability of each coin toss. According to Ariew, Rohwer, and Rice those two explanations cannot really be distinguished, and should both be regarded as statistical, non-causal explanations of the same fact. In their view, Lange's account is too influenced by his prior commitment to "causal-centric accounts of scientific explanation" (Ariew et al., 2017, p 71). Since whether those two statistical explanations can really be distinguished does not impinge on the claim that in statistical, non-causal explanations it is mathematics that is mainly doing the explaining, I will not take into account their criticism of Lange's view here. Contrary to this claim, it might be objected that autonomous-statistical explanations might be regarded as statistical, non-causal explanations that are not MENP. Indeed, according to Ariew, Rohwer, and Rice there can be an autonomous-statistical explanation for Galton's explanandum only if "the frequency distribution of the trait in the population is approximately normal. What determines the degree to which a real-world population approximates a normal distribution depends on minimal material requirements of the system" (ibidem, p. 64). This requirement might let someone think that autonomous-statistical explanations explain causal phenomena by interpreting them statistically, so that those explanations, albeit being statistical and non-causal, should not be regarded as genuine MENP. Indeed, "treating biological phenomena as mathematical (statistical) ones is not the same as conceiving of them as statistical phenomena in and of themselves" (Witteveen, 2019, p. 15). However, this interpretation has been convincingly contrasted by Witteveen (2019), who analyzes Ariew's, Rohwer's and Rice's proposal in the light of Galton's own works and reaffirms that "Galton's statistically autonomous explanation was not an explanation of a causal phenomenon treated statistically, but an explanation of a purely statistical phenomenon [...] in terms of another statistical phenomenon" (ibidem). If autonomous-statistical explanations are analogous to Galton's explanation, this means that autonomous-statistical explanations do not explain causal phenomena by interpreting them statistically. Rather, those explanations "explain by showing that what may initially appear to be a causal phenomenon is in fact a purely statistical effect" (ibidem, p. 16). So, it is fair to claim that autonomous-statistical explanations can be regarded as MENP. 


\section{Walsh's Account of Population Genetics Explanations}

To further illustrate how it might be difficult to recognize that statisticalists should address the challenge presented in this paper, in this section I analyse Walsh's account of population genetics explanations. I focus on Walsh's work, since he embraces statisticalism and his counterfactual account of the explanations provided by population genetics may appear to many readers as a good example of an account that is both non-causal and compatible with a naturalist stance. Since Walsh's account may thus represent a counterexample to what I said so far about the difficulty of combining statisticalism and naturalism, it is worth being discussed here in some detail. One of the reasons why the fact that statisticalists should address the challenge presented in this paper has so far gone unnoticed is that very often the way in which statisticalists present their ideas on population genetics explanations is not sufficiently explicit about the issue of whether or not statistical explanations in population genetics are genuine MENP. Statisticalists are indeed mainly devoted to defending the non-causal character of the explanations provided by population genetics, while they are usually less interested in addressing the issue of whether those non-causal explanations are distinctively mathematical, i.e., MENP. So, the difficulty of combining statisticalism with a naturalist stance might be not so easy to grasp at first.

It might be objected that if a commitment to statistical, non-causal explanations that are genuine MENP is constitutive of the statisticalist view, and naturalism is construed as implying a commitment to the unacceptability of MENP, it is not fair to accuse statisticalism of being incompatible with naturalism. To underline that statisticalism implies such a commitment would be no more than a restatement of the statisticalist position. From the statisticalist point of view, it might equally well be objected to naturalists that naturalism is untenable because it is not able to account for a genuine kind of non-causal, scientific explanations, namely MENP. So, the challenge here presented would be pointless. But the point I wish to make is precisely that usually statisticalists do not describe the acceptance of MENP as constitutive of their view, since they do not explicitly commit to MENP. Nor do they usually explicitly define themselves as anti-naturalists. Statisticalists are mainly interested in contrasting the reification of evolutive factors such as natural selection and drift in population genetics explanations, an aim that per se is not in contrast with a naturalist stance. Rather, the idea of excluding superfluous entities from one's ontology by inquiring whether explanations in a given domain are genuinely causal seems prima facie in line with a broadly construed naturalist view. So, I think that the challenge presented here is not pointless and that statisticalists should more directly address the concerns presented in this paper to better clarify their position.

Coming to Walsh's account, I intentionally leave aside the criticisms that have been moved to Walsh's account so far (e.g., Abrams, 2013; Northcott, 2010), since they do not deal with the issue I wish to focus on here. It is also worth clarifying that my analysis is not intended to be a criticism of Walsh's work in the philosophy of biology, which I appreciate, it just aims to show that more has to be done by the statisticalists in order to adequately address the challenge presented here.

\subsection{Drift Explanations as MENP}

According to Walsh (2015), there are genuine statistical, non-causal scientific explanations, and the explanations provided by theoretical population biology are explanations of this 
kind. Walsh tries to support this view by sketching an adequate conception of non-causal explanations. In order to reach his goal, Walsh moves from Woodward's interventionist account of explanation, which is usually understood to be able to provide an adequate account of causal explanations, and then he tries to extend this conception to cover noncausal explanations. This strategy has been adopted in recent years by several philosophers who aimed at providing an adequate account of non-causal explanations in different domains (see Ariew et al., 2015, for non-causal explanations in population genetics; Reutlinger, 2016, for non-causal explanations in metaphysics; Baron et al., 2017; 2019, for mathematical explanations; Reutlinger et al., 2020, for non-causal explanations in science and pure mathematics). All of those philosophers elaborate on Woodward's counterfactual theory of explanation, according to which explanation "is a matter of exhibiting systematic patterns of counterfactuals dependence" (Woodward, 2003, p. 191). In order to extend Woodward's counterfactual theory of explanation, those philosophers exploit a suggestion made by Woodward himself: the "common element in many forms of explanation, both causal and non-causal, is that they must answer what-if-things-had-been-different questions" (ibidem, p. 221). They so try to develop a counterfactual theory of explanation which might be able to account for both causal and non-causal explanations.

According to Walsh, an explanation serves two functions, the metaphysical and the cognitive function: the "metaphysical function is discharged by identifying a counterfactually robust invariance relation between explanans event and explanandum," while the "cognitive function is discharged by providing an appropriate description of this relation" (Walsh, 2015, p. 469). In Walsh's view, an explanation explains by showing that a change-relating invariance relation between the explanans and the explanandum holds. Change-relating invariance is a kind of counterfactual-supporting relation between the values of variables. A relation between $\mathrm{X}$ and $\mathrm{Y}$ is change-relating just if a difference in the value of $\mathrm{X}$ is associated with a difference in the value of $Y$ (Woodward, 2003). A relation between X and Y must also be invariant. Woodward defines an "invariant relation" in terms of intervention: a relation $R$, which relates the changes in the value of $X$ to changes in the value of $Y$, is invariant if $\mathrm{R}$ would continue to hold under some intervention that changes the value of $\mathrm{X}$ in such a way that, according to $\mathrm{R}$, the value of $\mathrm{Y}$ would change.

Walsh clarifies that intervention is used in Woodward's account of explanation merely as a diagnostic test for invariance, and not as part of its definition. So, despite intervention' is clearly a causal notion that, according to Woodward, guides us to the discovery of change-relating invariance relations, and despite paradigm cases of change-relating invariance relations are causal, the definition of change-relating invariance relation is not causal. And this "leaves open, at least as a conceptual possibility, that there might be other, noncausal, instances of change-relating invariance" relations (Walsh, 2015, p. 472). If there are such genuine non-causal change-relating invariance relations, they can well figure as explanantia in genuine non-causal explanations.

Now, when one deals with ordinary causal explanations, one has "to identify a set of conditions in the world-the explanans conditions-such that when they hold the explanandum does too" (ibidem, p. 471). But here we are dealing with the case of scientific, non-causal explanations, so, while the explanandum can be understood as a worldly phenomenon as it is in the case of causal explanations, it has to be specified to which "world" in this case the explanans conditions belong. Indeed, even if one concedes that the set of non-causal explanations and the set of distinctively mathematical explanations are not equal, the set of non-causal explanations is usually thought to include as a proper subset the set of distinctively mathematical explanations, i.e., the set of all MENP. Since mathematics is non-empirical, and non-empirical explanations are usually refused by 
naturalists, in order to clarify the issue we are dealing with, i.e., whether Walsh's view is really compatible with a naturalist stance, it should be explicitly stated whether or not the explanations in that proper subset of the set of non-causal explanations are acceptable in Walsh's view, and reason should be provided for why it is so.

To recapitulate, in Walsh's view, when one deals with non-causal explanations, one searches for a set of non-causal conditions $X$, such that when they hold, some empirical fact $\mathrm{Y}$ does hold too. Those non-causal conditions have also to be such that when one "manipulates" X, Y will invariantly change according to R. The point is whether in Walsh's view $\mathrm{X}$ and $\mathrm{R}$ can be mathematical in character.

Consider first the explanans conditions, i.e., X. Here Walsh faces a sort of dilemma: (1) either explanans conditions are non-causal and mathematical in character; or (2) they are non-causal and non-mathematical in character. If (1), i.e., the explanans conditions are mathematical, and so non-empirical, then Walsh's account of the explanations provided by population genetics perfectly fits MENP's description: there is some nonempirical explanans which allegedly explains an empirical explanandum. If this is the case, Walsh should provide in any case: (a) an account of how it is that we can acquire reliable knowledge of the mathematical conditions that constitute the explanans; and (b) an account of how it is that a set of mathematical conditions can explain some empirical fact. In addition to that, if Walsh wishes also to claim that his account of non-causal explanations is compatible with a naturalist stance, both (a) and (b) should be acceptable from a naturalist point of view. In other words, he should address the challenge described in this paper.

If (2), i.e., the explanans conditions are non-mathematical, then Walsh's account of the explanations provided by population genetics diverges from MENP's description. If this is the case, then Walsh should make in any case clearer what kind of conditions those noncausal and non-mathematical conditions are and defend the claim that those explanans conditions cannot be construed in causal terms by any account developed by the supporters of the causal view on explanation to show that there are no examples of genuine non-causal explanation of particular empirical facts. Indeed, as already noted, it seems that so far the only examples of non-causal explanations that have been adequately defended in the literature against the supporters of the causal view on explanation are all MENP (Reutlinger, 2017; Reutlinger \& Saatsi, 2018). At this regard, it is interesting to note that even the noncausality of explanations in statistical mechanics, traditionally regarded as scientific, noncausal explanations, has been fiercely disputed in recent years, to the extent that now philosophers regard the issue of whether all explanations in statistical mechanics are really non-causal as controversial. For example, Morrison (2018) argues that the non-causal nature of standard statistical mechanics explanations is disputed, so that in order to find some genuine instances of non-causal explanation in science it is better to focus on MENP, whose non-causality is less controversial, given that they are mathematical in character. Some philosophers, for instance, to defend the claim that some explanations in statistical mechanics are genuinely non-causal, focus on Renormalization Group (RG) explanations. They claim that RG explanations are non-causal precisely because RG explanations are MENP (Morrison, 2018; Reutlinger, 2018). So, again, it is difficult to find uncontroversial examples of non-causal and non-mathematical explanations in science.

Please, note that in order to claim that Walsh has not to address the present challenge it is not sufficient to show that some explanations in Walsh's account of population genetics explanations are not MENP. What should be demonstrated is that all the explanations regarded by Walsh as genuine populations genetics explanations are not MENP. Indeed, even if just some MENP are acceptable explanations in the light of Walsh's account of 
non-causal explanations, then Walsh should address the present challenge. The compatibility with a naturalist stance is not a matter of quantity. So, to investigate whether Walsh's account is compatible with a naturalist stance, we have to search whether at least one kind of MENP is an acceptable kind of explanations in Walsh's view.

And it seems that at least one kind of MENP is in fact acceptable in Walsh's view, namely drift explanations. Indeed, in several places Walsh describes drift explanations along the same lines in which drift explanations are described by MENP supporters, such as Lange (2013a). For example, in Walsh (2007) he explains why drift is stronger in smaller populations by making reference to different series of coin tosses and the CLT.

Walsh's example can be summarized as follows. Suppose that two fair coins are tossed 50 times each. The result of each toss can be either head $(\mathrm{H})$ or tail $(\mathrm{T})$. The probability of $\mathrm{H}$ and $\mathrm{T}$ "can be seen as the analogue of the fitnesses of two alleles at the same locus" (Walsh, 2007, p. 293), and the number of tosses can be seen as the analogue of the size of a population. There are at least three different ways to describe the 100 tosses performed.

(1) A single series of 100 tosses of a fair coin. The results are:

(a) $49 \mathrm{H} ; 51 \mathrm{~T}$

(2) Two series of 50 tosses of a fair coin, with two coins being tossed. In this case, the results are:

(a) $20 \mathrm{H} ; 30 \mathrm{~T}$

(b) $29 \mathrm{H} ; 21 \mathrm{~T}$

(3) Ten series of 10 tosses of a fair coin. The results are of the tosses are:

(a) $4 \mathrm{H} ; 6 \mathrm{~T}$

(b) $7 \mathrm{H} ; 3 \mathrm{~T}$

(c) $3 \mathrm{H} ; 7 \mathrm{~T}$

(d) $5 \mathrm{H} ; 5 \mathrm{~T}$

(e) $4 \mathrm{H} ; 6 \mathrm{~T}$

(f) $5 \mathrm{H} ; 5 \mathrm{~T}$

(g) $5 \mathrm{H} ; 5 \mathrm{~T}$

(h) $5 \mathrm{H} ; 5 \mathrm{~T}$

(i) $4 \mathrm{H} ; 6 \mathrm{~T}$

(j) $7 \mathrm{H} ; 3 \mathrm{~T}$

What is notable and worth of being explained is that in the "population" of 100 tosses taken as a whole, there is only a slight departure from the expected outcome of $50 \mathrm{H}$ and $50 \mathrm{~T}$. On the contrary, there is a considerably greater degree of departure from expectation in the population considered as two series of 50 tosses. Finally, the degree of departure from expectation observed is greater still when we consider the same population as ten series of 10 tosses. But those three results do not refer to three different populations, rather they are "different ways of describing the same population" (ibidem, p. 296). Now, according to Walsh, the only way to provide a satisfactory explanation of why the "force of drift in the population as a whole is small" (ibidem) is to deny that that population can be regarded as really made of ten independent tosses. Only if we consider it as a whole we can explain the fact that drift is larger in smaller sub-populations of a given population. Indeed, in this way we can regard each of the ten series of 10 tosses as "drawn at random from a larger, normally distributed population" (ibidem), and this would explain why drift is larger in the series of ten tosses that constitutes the population than in the population as a whole: it is the "central limit theorem [that] entails that the means of samples of the same size drawn from a normal 
distribution will themselves be normally distributed about the population mean" (ibidem). When the whole population is considered, "it is to be expected that the degree to which the outcomes in these subpopulations depart from the grand mean will tend to offset one another" (ibidem), and so it is to be expected that drift will be smaller in larger population.

Other similar passages might be quoted from different works by Walsh. For instance, in Walsh et al. (2002), drift is explained by making reference to the law of large numbers (Walsh et al., 2002, p. 459). Now, albeit Walsh does not explicitly claim that drift explanations are MENP, as already noted, it seems particularly transparent that in drift explanations some results from probability theory, i.e., some mathematical facts, are mainly doing the explaining, and so that drift explanations can be regarded as genuine MENP. Moreover, the way in which Walsh illustrates drift explanations is not in contrast with this interpretation of drift explanations as MENP.

\subsection{The Counterfactual Account of Explanation and MENP}

Turning back to Walsh's counterfactual account of explanation, in the case of drift explanation just discussed, it seems that, according to Walsh, the explanans conditions, i.e., X, is the CLT, i.e., a theorem from probability theory. So, it seems that X can be mathematical in character in Walsh's account of statistical, non-causal explanations. In the example just discussed, Y, i.e., the explanandum, is the fact that drift is smaller in the whole population than in sub-populations.

Consider now the relation that obtains between $\mathrm{X}$ and $\mathrm{Y}$, i.e., $\mathrm{R}$. $\mathrm{R}$ is a kind of counterfactual-supporting relation between the explanans and the explanandum. If $\mathrm{X}$ is a mathematical fact, $\mathrm{R}$ has to be a kind of counterfactual-supporting relation that can obtain between a mathematical fact and an empirical fact. We already mentioned the difficulty of developing an account of such a relation that might be acceptable for the naturalists. Here it is interesting to note that if drift explanations are really genuine MENP that are acceptable in Walsh's view, Walsh's attempt to account for population genetics explanations by developing a counterfactual account of explanation puts Walsh in contrast with the view on this issue defended by other two famous statisticalists, namely Matthen and Ariew (2009). Indeed, if drift explanations are MENP and one adopts a counterfactual account of explanation, one needs to counterfactually 'manipulate' mathematical facts. Matthen and Ariew, as well as Walsh, defend the claim that population genetics explanations are non-causal explanations. But Matthen and Ariew also explicitly claim that in those explanations the relation between the explanans and the explanandum, i.e., $\mathrm{R}$, is mathematical in character. And precisely for this reason they explicitly refuse the possibility that any counterfactual account of explanation can account for that kind of explanations, since they regard as impossible to counterfactually modify the mathematical facts that figure in those explanations. Matthen and Ariew clearly state that:

the theory of natural selection is different from that of gravitation, for natural selection is mathematically necessary. When there are heritable differences in traits leading to differential reproduction rates, the probability of the fitter types increasing in frequency is greater than that of the less-fit types increasing. This is simply a mathematical truth. Applying the manipulability test, we have to tolerate hypothetical interventions that are physically impossible, but refuse to tolerate hypothetical interventions that are mathematically impossible. (Matthen \& Ariew, 2009, p. 211, italics mine). 
So, it seems fair to claim that, according to Matthen and Ariew, populations genetics explanations are non-causal explanations precisely because they are mathematical in character, and so those explanations can be regarded as MENP. In their view, this very fact makes impossible to account for populations genetics explanations by the means of a counterfactual account of explanation. ${ }^{14}$ Cast in possible worlds terms, the problem is that, as noted above (Sect. 4), while it is usually assumed that possible worlds where any given law of nature does not hold are conceivable, it is instead widely assumed that it is not possible to conceive of any possible world where a mathematical truth does not hold. This means that in order to account for counterfactuals where "mathematically impossible" interventions are allowed, one has to commit oneself to a non-standard semantics for counterfactuals, i.e., a semantics that allows for metaphysically impossible worlds.

The point is that here Walsh faces a sort of dilemma: (1) either he wishes to contrast Matthen's and Ariew's perspective on population genetics explanations and defend the claim that in his view the statistical, non-causal explanations one finds in population genetics are not genuine MENP; or (2) he shares Matthen's and Ariew's view on the mathematical character of population genetics explanations, and so regard his statistical, non-causal explanations as genuine MENP.

If Walsh takes (1), in addition to the difficulties he has to face to prove that statistical explanations in population genetics are not MENP we already mentioned, he differentiates his view from that of Matthen and Ariew on a crucial point for the statisticalist position, namely the idea that population genetics explanations are mathematical in character, while so far no clue of such a theoretical distinction emerged in their writings, especially if one considers a recently published paper they coauthored, namely Walsh et al. (2017).

If instead Walsh takes (2), albeit he sticks to Matthen's and Ariew's view on the mathematical character of population genetics explanations, he nevertheless differentiates his position from that of Matthen and Ariew on another crucial point for the statisticalist position, namely what theory of explanation one should commit to in order to account for population genetics explanations, since Matthen and Ariew refuse a counterfactual account of explanation in the case of mathematical explanations in population genetics, i.e., in the case of MENP. It is interesting to note, albeit just in passing, that if Walsh takes this route, he should not only face the challenge presented in this paper because he accepts MENP in his view and MENP are unacceptable explanations from a naturalist point of view. He should also face the challenge here presented because of the counterfactual account of scientific explanation he commits to. Indeed, if one regards statistical, non-causal explanations in population genetics as genuine MENP, and commits oneself to a counterfactual account of explanation, in order to address the present challenge one has to find a way to make impossible worlds compatible with a naturalist stance. ${ }^{15}$

\footnotetext{
14 Whether one has to subscribe to the claim that a counterfactual theory of explanation cannot account for cases in which what has to be 'manipulated' belongs to the mathematical domain is a strongly debated issue. For a counterfactual account of MENP where impossible perturbations to mathematics are allowed, see Baron et al., 2017.

15 To appreciate the difficulty of providing a naturalist account of impossible worlds, please keep in mind the discussion made above about the fact that usually MENP supporters commit themselves to a realist perspective on modality (Sect. 6). As in the case of possible worlds, if several realist views on impossible worlds are available in the literature (for a survey, see Berto \& Jago, 2018, Sect. 3), they are usually thought to be in contrast with a naturalist stance. Unfortunately, this issue cannot be fully explored here for reason of space and has to be treated in a different paper.
} 


\section{Conclusions}

In this article, I argued that if the explanations provided by population genetics are regarded as non-causal explanations in which some mathematical fact is indispensably doing the explaining, then the explanations provided by population genetics have to be regarded as MENP. I also argued that it is not easy to combine MENP with naturalism. If what I said is sound, it seems fair to conclude by saying that the burden of proof that either (1) the explanations provided by population genetics are not really MENP and are compatible with a naturalist stance, or (2) MENP are not really incompatible with a naturalist stance, is on the statisticalist who wishes to profess herself to be a naturalist.

Funding Open access funding provided by Università degli Studi di Roma La Sapienza within the CRUICARE Agreement.

\section{Declarations}

Conflicts of interest I declare that I have no conflict of interest.

Open Access This article is licensed under a Creative Commons Attribution 4.0 International License, which permits use, sharing, adaptation, distribution and reproduction in any medium or format, as long as you give appropriate credit to the original author(s) and the source, provide a link to the Creative Commons licence, and indicate if changes were made. The images or other third party material in this article are included in the article's Creative Commons licence, unless indicated otherwise in a credit line to the material. If material is not included in the article's Creative Commons licence and your intended use is not permitted by statutory regulation or exceeds the permitted use, you will need to obtain permission directly from the copyright holder. To view a copy of this licence, visit http://creativecommons.org/licenses/by/4.0/.

\section{References}

Abrams, M. (2013). Populations and Pigeons: Prosaic Pluralism about Evolutionary Causes. Studies in the History and Philosophy of Biology and the Biomedical Sciences, 44, 294-301.

Andersen, H. (2018). Complements, Not Competitors: Causal and Mathematical Explanations. The British Journal for the Philosophy of Science, 69, 485-508.

Ariew, A., Rice, C., \& Rohwer, Y. (2015). Autonomous-Statistical Explanations and Natural Selection. The British Journal for the Philosophy of Science, 66, 635-658.

Ariew, A., Rohwer, Y., \& Rice, C. (2017). Galton, Reversion and the Quincunx: The Rise of statistical Explanation. Studies in History and Philosophy of Biological and Biomedical Sciences, 66, 63-72.

Baker, A. (2016). Simplicity. In E. N. Zalta (Ed.), The Stanford Encyclopedia of Philosophy. https://plato. stanford.edu/archives/win2016/entries/simplicity/.

Baker, A. (2009). Mathematical Explanation in Science. The British Journal for the Philosophy of Science, $60,611-633$.

Balaguer, M. (2009). Realism and Anti-Realism in Mathematics. In D. Gabbay, P. Thagard, \& J. Woods (Eds.), Handbook of the Philosophy of Science (Vol. 4, pp. 117-151). Elsevier.

Baron, S. (2014). Optimisation and Mathematical Explanation: Doing the Lévy Walk. Synthese, 191, 459-479.

Baron, S. (2015). Mathematical Explanation and Epistemology: Please Mind the Gap. Ratio, 29, 149-167.

Baron, S., Colyvan, M., \& Ripley, D. (2017). How Mathematics Can Make a Difference. Philosophers' Imprint, 17, 1-19.

Baron, S., Colyvan, M., \& Ripley, D. (2019). A Counterfactual Approach to Explanation in Mathematics. Philosophia Mathematica. https://doi.org/10.1093/philmat/nkz023.

Benacerraf, P. (1973). Mathematical Truth. The Journal of Philosophy, 70, 661-679.

Berenstain, N. (2017). The Applicability of Mathematics to Physical Modality. Synthese, 194, 3361-3377. 
Berto, F., \& Jago, M. (2018). Impossible Worlds. In E. N. Zalta (Ed.), The Stanford Encyclopedia of Philosophy. https://plato.stanford.edu/archives/fall2018/entries/impossible-worlds/.

Bertrand, M. (2018). Metaphysical Explanation by Constraint. Erkenntnis. https://doi.org/10.1007/ s10670-018-0009-5.

Blanchard, T. (2016). Physics and Causation. Philosophy Compass, 11, 256-266.

Bokulich, A. (2018). Searching for Non-Causal Explanations in a Sea of Causes. In A. Reutlinger \& J. Saatsi (Eds.), Explanation Beyond Causation (pp. 141-163). Oxford University Press.

Brown, J. R. (2012). Platonism, Naturalism, and Mathematical Knowledge. Routledge.

Bueno, O. (2011). Logical and Mathematical Knowledge. In S. Bernecker \& D. Pritchard (Eds.), The Routledge Companion to Epistemology (pp. 358-368). Routledge.

Bueno, O. (2017). The Epistemology of Modality and the Epistemology of Mathematics. In B. Fischer \& F. Leon (Eds.), Modal Epistemology After Rationalism (pp. 67-83). Springer.

Colyvan, M. (2019). Indispensability Arguments in the Philosophy of Mathematics. In E. N. Zalta (Ed.), The Stanford Encyclopedia of Philosophy. https://plato.stanford.edu/archives/spr2019/entries/mathp hil-indis/.

Cramer, H. (1946). Mathematical Methods of Statistics. Princeton University Press.

Devitt, M. (1998). Naturalism and the A Priori. Philosophical Studies, 92, 45-65.

Field, H. (1989). Realism, Mathematics, and Modality. Basil Blackwell.

French, S. (2014). The Structure of the World. Oxford University Press.

Giere, R. N. (2006). Modest Evolutionary Naturalism. Biological Theory, 1, 52-60.

Glazier, M. (2017). The Difference between Epistemic and Metaphysical Necessity. Synthese. https://doi. org/10.1007/s11229-017-1626-2.

Gödel, K. (1990). Collected Works (Vol. 2). Oxford University Press.

Gödel, K. (1995). Collected Works (Vol. 3). Oxford University Press.

Hales, T. C. (2001). The Honeycomb Conjecture. Discrete and Computational Geometry, 25, 1-22.

Hempel, C. G., \& Oppenheim, P. (1948). Studies in the Logic of Explanation. Philosophy of Science, 15, 135-175.

Hitchcock, C., \& Velasco, J. (2014). Evolutionary and Newtonian Forces. Ergo, 1, 39-77.

Honerkamp, J. (1983). The Heart as a System of Coupled Nonlinear Oscillators. Journal of Mathematical Biology, 18, 69-88.

Horsten, L. (2015). Philosophy of Mathematics. In E. N. Zalta (Ed.), The Stanford Encyclopedia of Philosophy. http://plato.stanford.edu/archives/spr2015/entries/philosophy-mathematics/.

Huneman, P. (2010). Topological Explanations and Robustness in Biological Sciences. Synthese, 177, 213-245.

Huneman, P. (2018). Diversifying the Picture of Explanations in Biological Sciences: Ways of Combining Topology with Mechanisms. Synthese, 195, 115-146.

Jonas, S. (2017). Access Problems and Explanatory Overkill. Philosophical Studies, 174, 2731-2742.

Lange, M. (2013a). Really Statistical Explanations and Genetic Drift. Philosophy of Science, 80, $169-188$.

Lange, M. (2013b). What Makes a Scientific Explanation Distinctively Mathematical? The British Journal for the Philosophy of Science, 64, 485-511.

Lange, M. (2017). Because Without Cause. Oxford University Press.

Leng, M. (2007). Introduction. In M. Leng, A. Paseau, \& M. Potter (Eds.), Mathematical Knowledge (pp. 1-15). Oxford University Press.

Lewis, D. (1973). Counterfactuals. Blackwell.

Lewis, D. (1986). Philosophical Papers (Vol. 2). Oxford University Press.

Lipton, P. (2004). Inference to the Best Explanation (2nd ed.). Routledge.

Luque, V. J. (2016). Drift and Evolutionary Forces: Scrutinizing the Newtonian Analogy. Theoria, 31, $397-410$.

Lyon, A. (2014). Why Are Normal Distributions Normal? The British Journal for the Philosophy of Science, 65, 621-649.

Maddy, P. (1997). Naturalism in Mathematics. Oxford University Press.

Mancosu, P. (2018). Explanation in Mathematics. In E. N. Zalta (Ed.), The Stanford Encyclopedia of Philosophy. https://plato.stanford.edu/archives/sum2018/entries/mathematics-explanation/.

Matthen, M. (2010). What Is Drift? A Response to Millstein, Skipper, and Dietrich. Philosophy and Theory in Biology, 2, e102.

Matthen, M., \& Ariew, A. (2009). Selection and Causation. Philosophy of Science, 76, 201-224.

McEvoy, M. (2018). Apriority, Necessity and the Subordinate Role of Empirical Warrant in Mathematical Knowledge. Theoria, 84, 157-178. 
Millstein, R., Skipper, R. A. J., \& Dietrich, M. R. (2009). (Mis)interpreting Mathematical Models: Drift as a Physical Process. Philosophy and Theory in Biology, 1, e002.

Morrison, M. (2018). The Non-Causal Character of Renormalization Group Explanations. In A. Reutlinger \& J. Saatsi (Eds.), Explanation Beyond Causation (pp. 206-227). Oxford University Press.

Nolan, D. (2017). Naturalised Modal Epistemology. In B. Fischer \& F. Leon (Eds.), Modal Epistemology After Rationalism (pp. 7-27). Springer.

Northcott, R. (2010). Walsh on Causes and Evolution. Philosophy of Science, 77, 457-467.

Nozick, R. (2001). Invariances. Harvard University Press.

Otsuka, J. (2016). A Critical Review of the Statisticalist Debate. Biology \& Philosophy, 31, 459-482.

Papineau, D. (2016). Naturalism. In E. N. Zalta (Ed.), The Stanford Encyclopedia of Philosophy. https:// plato.stanford.edu/archives/win2016/entries/naturalism/.

Paseau, A. (2013). Naturalism in the Philosophy of Mathematics. In E. N. Zalta (Ed.), The Stanford Encyclopedia of Philosophy. https://plato.stanford.edu/archives/win2016/entries/naturalism-mathematics

Peacocke, C. (1999). Being Known. Clarendon Press.

Pincock, C. (2007). A Role for Mathematics in the Physical Sciences. Nô̂s, 41, 253-275.

Pincock, C. (2012). Mathematics and Scientific Representation. Oxford University Press.

Putnam, H. (1971). Philosophy of Logic. Harper and Row.

Quine, W. V. O. (1960). Word and Object. The MIT Press.

Reutlinger, A. (2016). Does the Counterfactual Theory of Explanation Apply to Non-Causal Explanations in Metaphysics? European Journal for Philosophy of Science, 7, 239-256.

Reutlinger, A. (2017). Explanation beyond Causation? New Directions in the Philosophy of Scientific Explanation. Philosophy Compass, 12, e12395. https://doi.org/10.1111/phc3.12395.

Reutlinger, A. (2018). Extending the Counterfactual Theory of Explanation. In A. Reutlinger \& J. Saatsi (Eds.), Explanation Beyond Causation (pp. 74-95). Oxford University Press.

Reutlinger, A., \& Andersen, H. (2016). Abstract versus Causal Explanations? International Studies in the Philosophy of Science, 30, 129-146.

Reutlinger, A., Colyvan, M., \& Krzyżanowska, K. (2020). The Prospects for a Monist Theory of Non-Causal Explanation in Science and Mathematics. Erkenntnis. https://doi.org/10.1007/s10670-020-00273-w.

Reutlinger, A., \& Saatsi, J. (Eds.). (2018). Explanation Beyond Causation. Oxford University Press.

Rice, C. (2015). Moving beyond Causes: Optimality Models and Scientific Explanation. Nô̂s, 49, 589-615.

Ryzhii, E., \& Ryzhii, M. (2014). Modeling of Heartbeat Dynamics with a System of Coupled Nonlinear Oscillators. In T. D. Pham, K. Ichikawa, M. Oyama-Higa, D. Coomans, \& X. Jiang (Eds.), Biomedical Informatics and Technology (pp. 67-75). Springer.

Salmon, W. C. (1971). Statistical Explanation. In W. C. Salmon, R. C. Jeffrey, \& J. G. Greeno (Eds.), Statistical Explanation and Statistical Relevance (pp. 29-87). University of Pittsburgh Press.

Salmon, W. C. (1977). A Third Dogma of Empiricism. In R. Butts \& J. Hintikka (Eds.), Basic Problems in Methodology and Linguistics (pp. 149-166). Reidel.

Salmon, W. C. (1989). Four Decades of Scientific Explanation. In P. Kitcher \& W. C. Salmon (Eds.), Scientific Explanation (pp. 3-219). University of Minnesota Press.

Shapiro, S. (1997). Philosophy of Mathematics. Oxford University Press.

Skow, B. (2014). Are There Non-Causal Explanations (of Particular Events)? The British Journal for the Philosophy of Science, 65, 445-467.

Sober, E. (1984). The Nature of Selection. The University of Chicago Press.

Stalnaker, R. (1968). A Theory of Conditionals. In N. Rescher (Ed.), Studies in Logical Theory (pp. 98-112). Blackwell.

Steiner, M. (1998). The Applicability of Mathematics as a Philosophical Problem. Harvard University Press. Stephens, C. (2004). Selection, Drift, and the 'Forces' of Evolution. Philosophy of Science, 71, 550-570.

Strevens, M. (2008). Depth. Harvard University Press.

Tahko, T. E. (2012). Counterfactuals and Modal Epistemology. Grazer Philosophische Studien, 86, 93-115.

Tegmark, M. (2008). The Mathematical Universe. Foundations of Physics, 38, 101-150.

Vaidya, A. (2016). The Epistemology of Modality. In E. N. Zalta (Ed.), The Stanford Encyclopedia of Philosophy. https://plato.stanford.edu/archives/win2016/entries/modality-epistemology/.

van Fraassen, B. C. (1989). Laws and Symmetry. Oxford University Press.

Walsh, D. M. (2007). The Pomp of Superfluous Causes: The Interpretation of Evolutionary Theory. Philosophy of Science, 74, 281-303.

Walsh, D. M. (2015). Variance, Invariance and Statistical Explanation. Erkenntnis, 80, 469-489.

Walsh, D. M., Ariew, A., \& Matthen, M. (2017). Four Pillars of Statisticalism. Philosophy, Theory, and Practice in Biology, 9, 1-18. 
Walsh, D. M., Lewens, T., \& Ariew, A. (2002). The Trials of Life: Natural Selection and Random Drift. Philosophy of Science, 69, 452-473.

Williamson, T. (2007). The Philosophy of Philosophy. Oxford University Press.

Witteveen, J. (2019). Regression Explanation and Statistical Autonomy. Biology \& Philosophy, 34, 1-20.

Woodward, J. (2017). Scientific Explanation. In E. N. Zalta (Ed.), The Stanford Encyclopedia of Philosophy. https://plato.stanford.edu/archives/fall2017/entries/scientific-explanation/.

Woodward, J. (2003). Making Things Happen. Oxford University Press.

Publisher's Note Springer Nature remains neutral with regard to jurisdictional claims in published maps and institutional affiliations.

Fabio Sterpetti is fixed-term assistant professor in Logic and Philosophy of Science at Sapienza University of Rome, Italy. He received his PhD in Philosophy from Sapienza University of Rome in 2012. His research focuses on the realism/anti-realism debate in philosophy of science and on the difficulty of making scientific realism compatible with a naturalist stance. His articles have appeared in journals such as Synthese, History and Philosophy of the Life Sciences, Philosophia, Axiomathes and Topoi. He co-edited, with Marta Bertolaso, the volume A Critical Reflection on Automated Science (Springer, 2020), and with Emiliano Ippoliti and Thomas Nickles, the volume Models and Inferences in Science (Springer, 2016). 